\title{
Sawflies (Hymenoptera, Symphyta) newly recorded from Washington State
}

\author{
Chris Looney', David R. Smith², Sharon J. Collman³ \\ David W. Langor ${ }^{4}$, Merrill A. Peterson ${ }^{5}$
}

I Washington State Dept. of Agriculture, 1111 Washington St. SE, Olympia, Washington, 98504, USA 2 Systematic Entomology Laboratory, Agricultural Research Service, USDA, clo National Museum of Natural History, NHB 168, Washington, D.C. 20560, USA 3 Washington State University Extension, 600 128th St. SE, Everett, Washington, 98208, USA 4 Natural Resources Canada, Canadian Forest Service, 5320122 Street NW, Edmonton, Alberta, T6H 3S5, Canada 5 Biology Department, Western Washington University, 516 High St., Bellingham, Washington, 98225, USA

Corresponding author: Chris Looney (clooney@agr.wa.gov)

$\frac{\text { Academic editor: H. Baur | Received } 5 \text { November } 2015 \text { | Accepted 27 January } 2016 \text { | Published } 28 \text { April } 2016}{\text { http://zoobank.org/319E4CAA-6B1F-408D-8A84-E202E14B26FC }}$

Citation: Looney C, Smith DR, Collman SJ, Langor DW, Peterson MA (2016) Sawflies (Hymenoptera, Symphyta) newly recorded from Washington State. Journal of Hymenoptera Research 49: 129-159. doi: 10.3897/JHR.49.7104

\begin{abstract}
Examination of museum specimens, unpublished collection data, and field surveys conducted between 2010 and 2014 resulted in records for 22 species of sawflies new to Washington State, seven of which are likely to be pest problems in ornamental landscapes. These data highlight the continued range expansion of exotic species across North America. These new records also indicate that our collective knowledge of Pacific Northwest arthropod biodiversity and biogeography is underdeveloped, even for a relatively well known and species-poor group of insects. Notable gaps in the knowledge of Washington State's Symphyta remain for the Olympic Peninsula, the Cascade Mountain Range, and the arid interior of the state. Washington's shrub-steppe appears to be particularly poorly surveyed for sawflies.
\end{abstract}

\section{Keywords}

Exotic species, range expansion, state record, museum data

Copyright Chris Looney et al. This is an open access article distributed under the terms of the Creative Commons Attribution License (CC BY 4.0), which permits unrestricted use, distribution, and reproduction in any medium, provided the original author and source are credited. 


\section{Introduction}

Sawflies (Hymenoptera, Symphyta) comprise 14 families worldwide, with 12 of these and about 1,000 described species known from North America (Smith 1979a, Taeger et al. 2010). Eleven families and approximately 180 named species are currently recorded from Washington State (Smith 1979a, Gibson 1980, Goulet 1986, Smith 1989, Goulet 1996, Looney et al. 2012, Schiff et al. 2012). Most sawfly species are herbivores, including leaf- and stem-mining species, chewing defoliators, wood-borers, and leaf-tying defoliators. Species in the family Orussidae are external parasitoids of wood-boring insects (Powell and Turner 1975, Deyrup 1984), and adult Tenthredo (Tenthredinidae) are commonly observed feeding upon other arthropods (Pasteels and Gregoire 1984). Some sawflies are important forestry, horticultural and agricultural pests.

The 2009 discovery of the introduced alder-feeding sawfly Monsoma pulveratum (Retzius, 1783) in the Pacific Northwest (Looney et al. 2012) provided impetus to conduct a broad sawfly survey in Washington State from 2010 through 2012. Specimens collected during that survey revealed that the Pacific Northwest range of many sawfly species is incompletely delineated, and that relatively few contributions have been made towards understanding Pacific Northwest Symphyta during recent decades. Subsequent to the two year survey, we collected data from other researchers, museum specimens, and further serendipitous discoveries. Here we report 22 species not previously known from Washington or documented only in gray literature, expanding known ranges in some cases and filling gaps in others.

\section{Methods}

New sawfly records were compiled from many sources, including regional entomological collections and recent field surveys. More than 3,500 identified and unidentified sawflies in entomology collections at the Evergreen State College, the College of Idaho, Oregon State University, the University of Idaho, Washington State University, and Western Washington University were examined for species of interest.

Field surveys from 2010 through 2012 employed sticky traps and Malaise traps (Fig. 1), with subsequent targeted and opportunistic hand collections made through 2015. Double-sided yellow, green, or white sticky traps with hot-applied adhesive were placed in various woody host plants across Washington in 2010-2012. Host material surveyed included alder, poplar, hawthorn, mountain ash, cherry, pear, apple, elderberry, and various conifers, with approximately 105 sites surveyed in Washington. Nine Malaise traps were installed in Washington west of the Cascade mountain range. Three traps were installed near ports of entry, two along the Columbia River, two in mixed-use forest stands, and two in residential areas. One Malaise trap was installed in a prairie remnant surrounded by agricultural fields in eastern Washington. Traps were installed in April, 2012, and maintained through September 2012. Opportunistic 


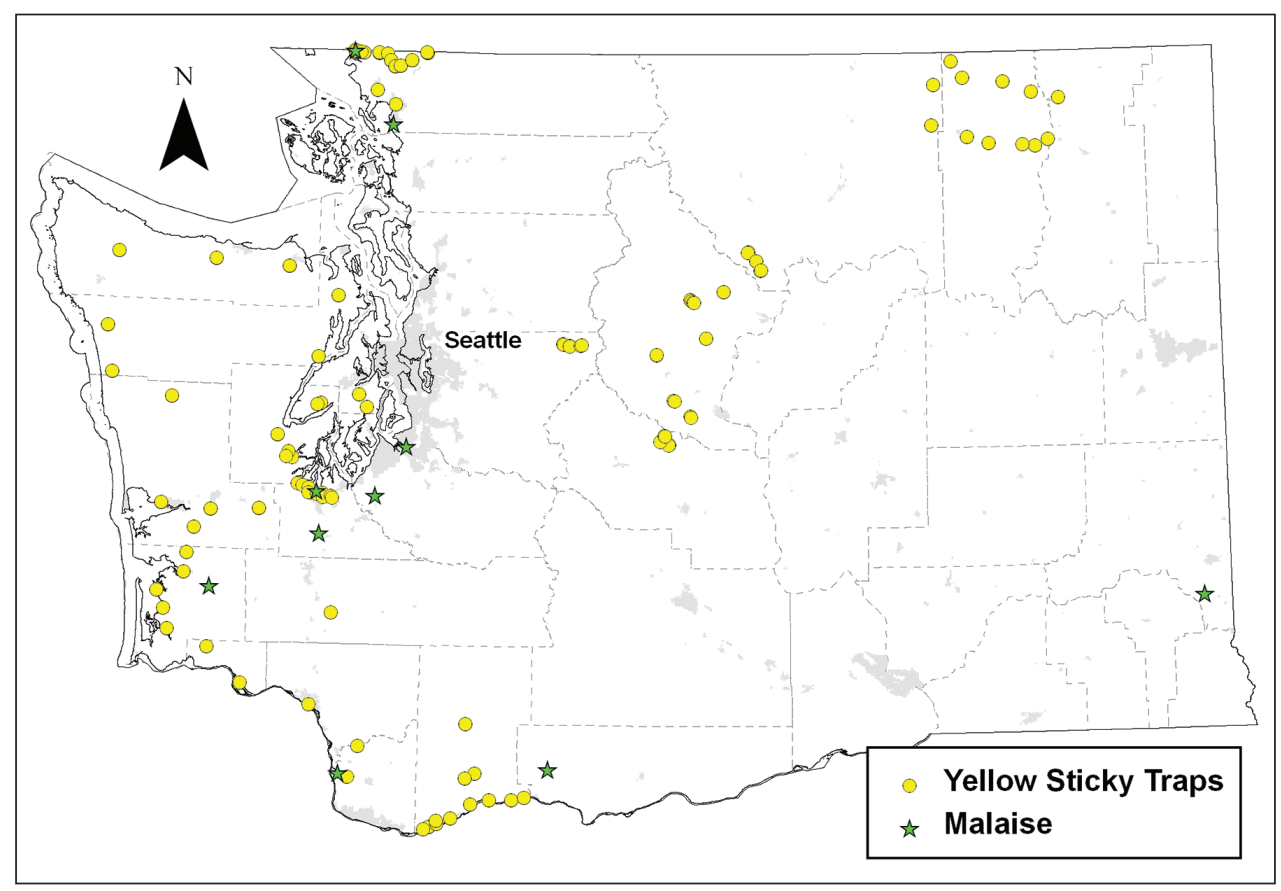

Figure I. Locations of yellow sticky traps and Malaise traps deployed in Washington State in 2011.

hand collections of larvae and adults were made throughout 2010-2015 as part of this study. We also present novel data for leaf-mining sawflies in Washington collected during a survey conducted in 2006 (see also Digweed et al. 2009). Lastly, some of the species recorded here were first detected by citizens reporting new pest problems (e.g., Neodiprion sertifer).

Collection data were compiled for each species, and narratives were composed that briefly describe each species' natural history and other details. Species names follow Taeger et al. (2010). We have chosen to not alter two combinations in the Nematinae suggested by new work by Prous et al. (2014). Taxonomic changes for the North American fauna resulting from their research have not yet been made, and are best approached via a thorough examination of North American Nematinae rather than piecemeal in papers such as this. Probable combinations are noted in the narrative accompanying each species. There are numerous common names ascribed to many of the species reported on here, since so many of these species are pestiferous and conspicuous. We do not provide those names, but North American common names can be found at the websites for the Entomological Society of Canada (esc-sec.ca) and the Entomological Society of America (entsoc.org). Voucher specimens are deposited at the Northern Forestry Centre Arthropod Collection in Edmonton, Alberta (NFRC), the Washington State Department of Agriculture Collection (WSDAC), Western Washington University (WWUC), the Evergreen State College (TESC), and the National Museum of Natural History, Smithsonian Institution, Washington, DC (USNM). 


\section{Results}

Twenty-two species not previously documented in Washington State in peer-reviewed literature were detected in these field and museum surveys, primarily in western Washington (Table 1). One species was collected as by-catch in a survey for other pests, and seven species were first detected due to citizen complaints. Five species were first detected by examining unidentified material in museum collections, and the remainder were collected in sawfly surveys or general collecting. A map of collection localities for more than 1,200 sawfly specimens indicates that most collecting has been near developed towns and cities, or along major highways (Fig. 2). Remote and rugged areas are undersampled, and very few collections have been made in the arid interior shrub-steppe.

\section{Xiphydriidae}

\section{Xiphydria prolongata (Geoffroy, 1785)}

Xiphydria prolongata is a European species that is a wood-borer in small limbs of deciduous tree genera, including Salix L., Quercus L., and numerous Betulaceae (Smith 1983). Larvae generally bore in decaying wood, and are not pestiferous. This western European species was first documented in North America in Michigan and New Jersey in the early 1980s, bringing the known Xiphydria species in North America to nine (Smith 1983). Mudge et al. (2001) recorded the first west coast specimen from Multnomah County, Oregon, in 1989. The single specimen from Washington was found in a trap for Japanese beetle, near Boeing Field in King County (Fig. 3). The specimen is housed at WSDAC.

\section{Diprionidae}

\section{Neodiprion sertifer (Geoffroy, 1785)}

A member of Ross's (1955) sertifer species group, this species is the only Neodiprion native to Europe, where it is a forestry and nursery pest (Day and Leather 1997). Its primary hosts are Pinus resinosa Aiton and P. sylvestris L., although it will feed on most Pinus species and can be a significant source of damage in pine plantations during outbreak years (Alford 2012). Introduced to New Jersey, USA, in 1925 (Schaffner 1939), its more visible impacts have included feeding damage on Christmas trees, making them less valuable or unmarketable, and defoliation of older needles on ornamental and landscape plants (Wilson 1971). Economic damage in North America has been recorded on P. strobus L., P. sylvestris, and P. mugo Turra, well as several native North American Pinus spp. (Schaffner 1943, Craighead 1950, Benjamin et al. 1955, Griffiths 1959, Baker 1972). Since its introduction, the species has spread westward at least to North Dakota (Van Driesche et al. 2012) and Saskatchewan, Canada (Langor, unpublished data). 


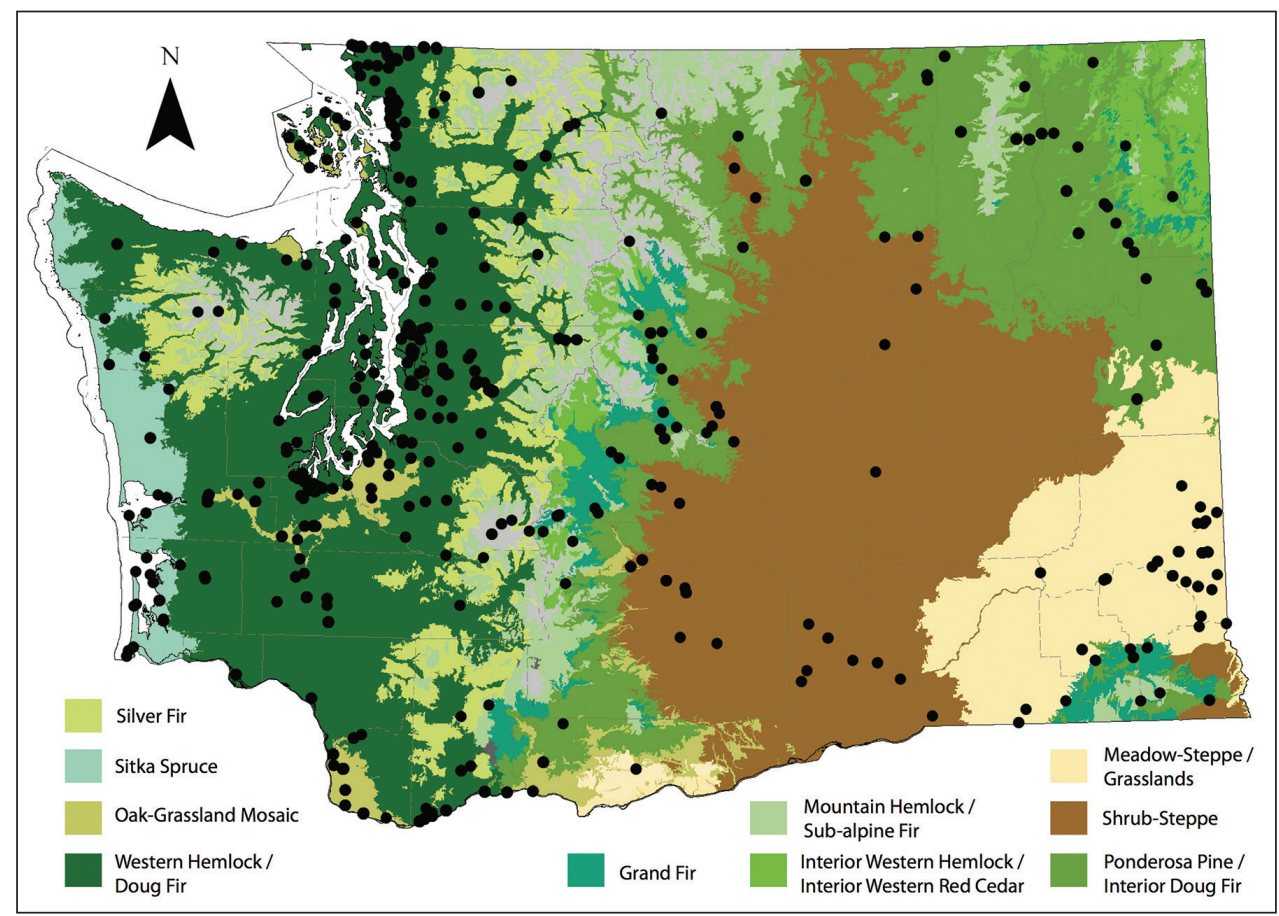

Figure 2. Map of specimen localities (black circles) for over 1,200 specimens collected in Washington State, including museum data and field data generated during this project. Vegetation zones are simplified from Washington's GAP Analysis project (Cassidy et al. 1997).

Neodiprion sertifer was detected in Washington State in 2008, when citizens in Bellingham, Washington, alerted pest control professionals to several defoliating outbreaks on ornamental pines. Larvae were observed again in 2012 on P. sylvestris and $P$. mugo in Bellingham, and adult males of Neodiprion sp. were collected in pheromone traps in the city that year. Although the latter were likely $N$. sertifer, male Neodiprion are not readily identifiable to species using external or genitalic morphology, or by mitochondrial DNA sequences (Linnen and Farrel 2012). Despite extensive surveys in 2010-2013, N. sertifer populations have to date only been found in Washington State within the Bellingham city limits (Fig. 3). The records from Washington State could represent a newer and separate introduction event in the Pacific Northwest, although transport on nursery stock seems to be the most likely introduction pathway. Specimens are housed at WSDAC.

\section{Diprion similis (Hartig, 1836)}

Taeger et al. (2010) list 13 world species of Diprion. Most are Asian, but D. similis is one of two Diprion species native to Europe. Large populations of this solitary feeder 


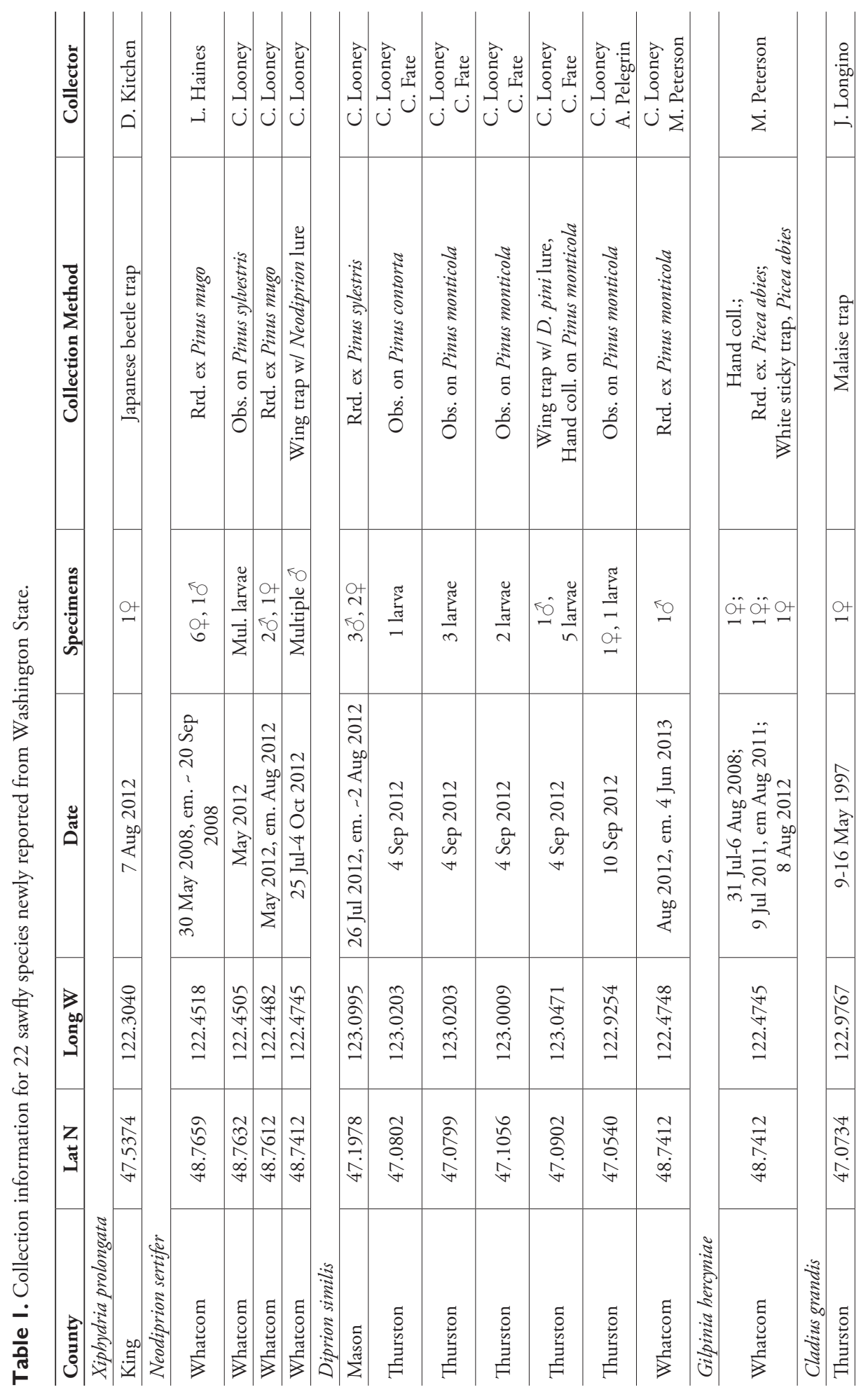




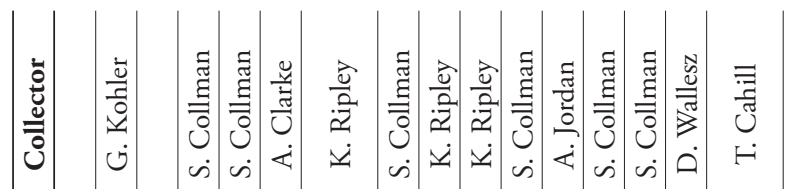

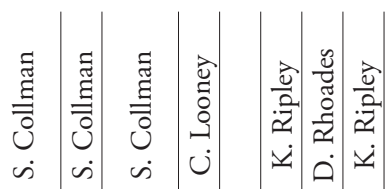

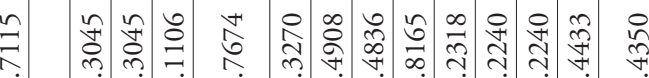




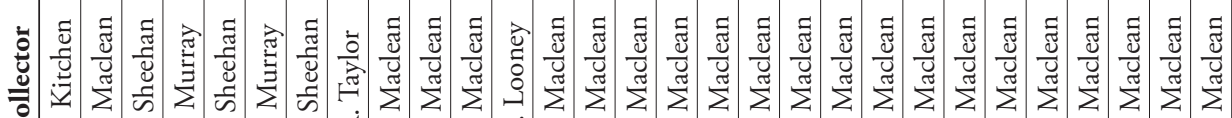

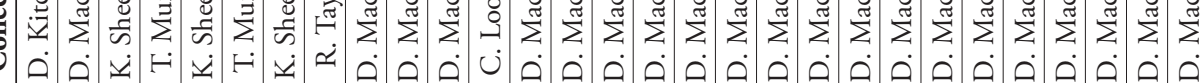

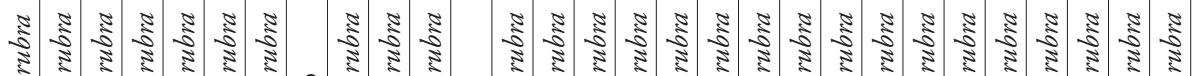

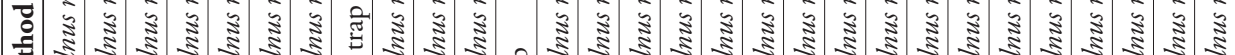

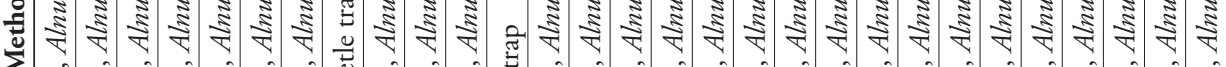

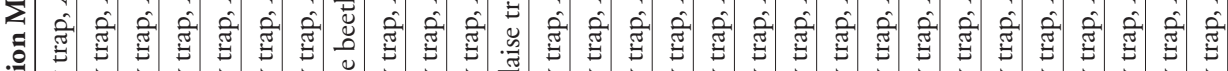

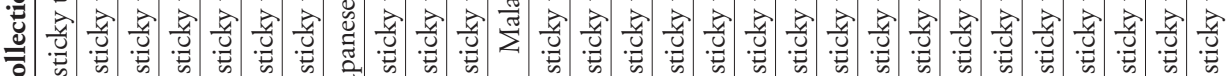

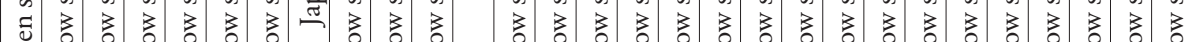

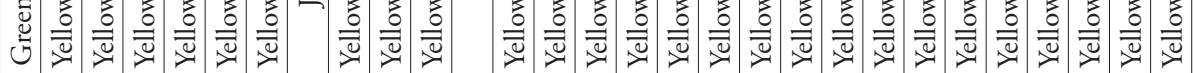

$\stackrel{\mathscr{Z}}{\tilde{d}}$

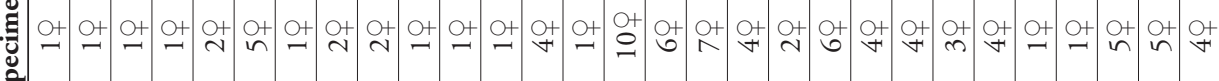

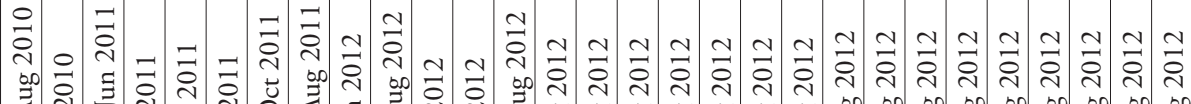
: ⿹勹巳

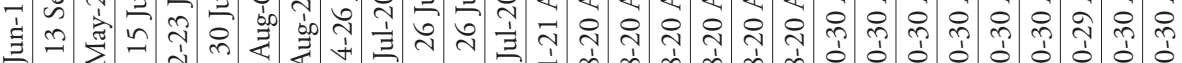

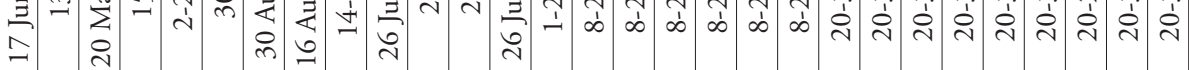

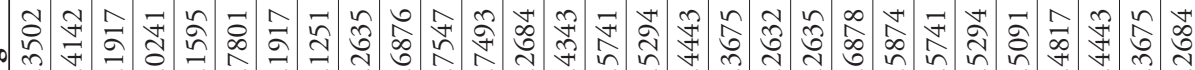

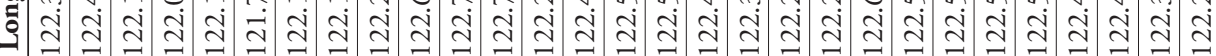

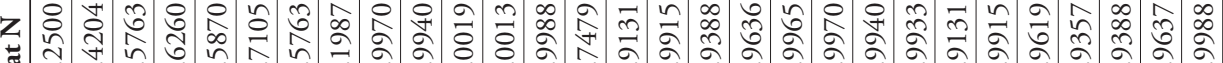

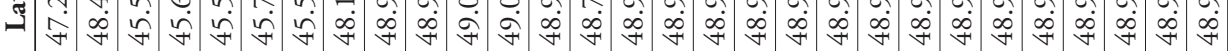

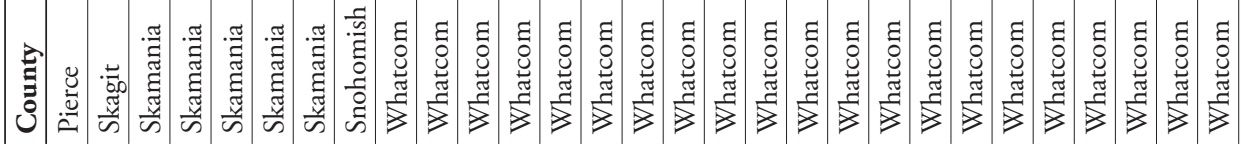




\begin{tabular}{|c|c|c|c|c|c|c|c|c|c|c|c|c|c|c|c|c|c|c|c|c|c|c|c|}
\hline 苞 & $\begin{array}{l}\text { है } \\
\overline{\bar{g}} \\
\text { v }\end{array}$ & $\begin{array}{l}\text { ज్ } \\
\text { है } \\
\overline{0} \\
\text { نे }\end{array}$ & 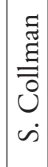 & 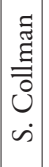 & 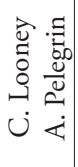 & 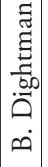 & $\begin{array}{c}\bar{\Xi} \\
\vdots \\
0 \\
. \\
ن \\
ن\end{array}$ & 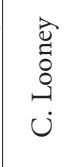 & 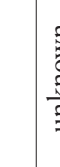 & 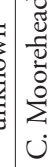 & & 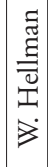 & 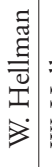 & 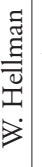 & $\begin{array}{l}\frac{\tilde{J}}{\tilde{J}} \\
\frac{\tilde{m}}{\tilde{u}} \\
\dot{0} \\
\dot{0}\end{array}$ & 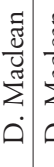 & | & 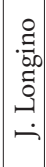 & 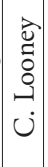 & $\begin{array}{l}0 \\
0 \\
0 \\
0 \\
ن \\
ن\end{array}$ & 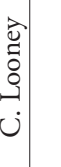 & 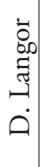 & 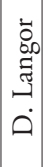 \\
\hline 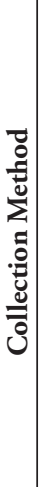 & 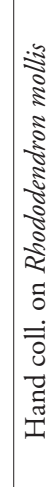 & 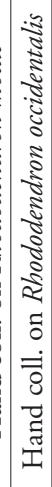 & 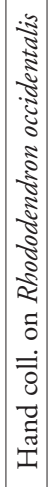 & 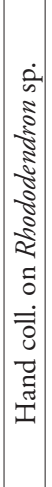 & 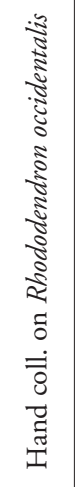 & 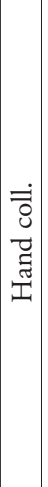 & 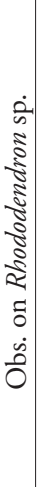 & 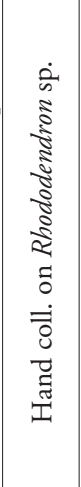 & 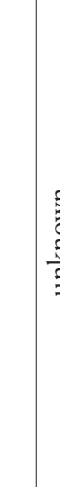 & 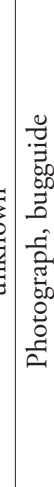 & & 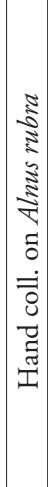 & 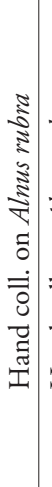 & 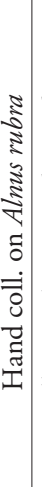 & 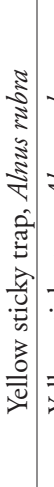 & 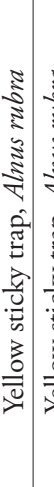 & 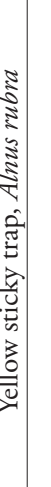 & 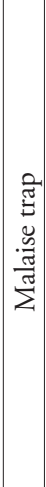 & 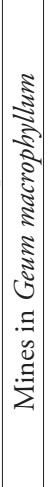 & 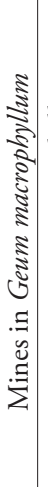 & & 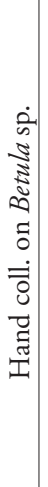 & 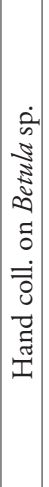 \\
\hline 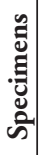 & & 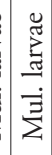 & 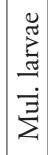 & 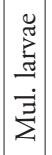 & 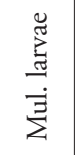 & $\stackrel{\circ}{-}$ & 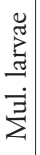 & 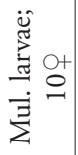 & O & 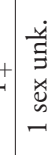 & & $\mid \begin{array}{c}\tilde{a} \\
\stackrel{\Xi}{a} \\
- \\
-\end{array}$ & $\begin{array}{l}\tilde{a} \\
\stackrel{a}{2} \\
-\end{array}$ & 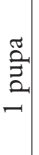 & $\begin{array}{c}0+ \\
= \\
r o \\
m \\
m\end{array}$ & 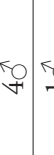 & -1 & $\stackrel{\mathrm{O}+}{-}$ & 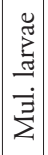 & 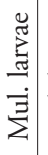 & 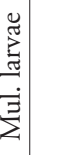 & 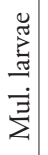 & 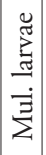 \\
\hline صै & 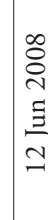 & $\begin{array}{l}\infty \\
\vdots \\
\vdots \\
\vdots \\
\vdots \\
a\end{array}$ & 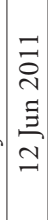 & $\left|\begin{array}{c}n \\
\stackrel{2}{2} \\
\Xi \\
\Xi\end{array}\right|$ & 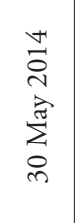 & 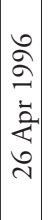 & 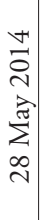 & 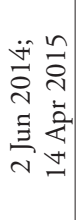 & ? & 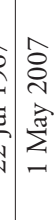 & & $\begin{array}{l}\vec{z} \\
\tilde{D} \\
2 \\
\tilde{\omega} \\
\sim \\
v\end{array}$ & 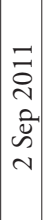 & 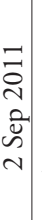 & 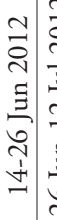 & 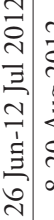 & 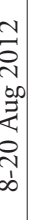 & 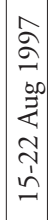 & 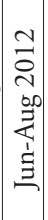 & $\begin{array}{l}\frac{ \pm}{D} \\
\stackrel{N}{\Xi} \\
\Xi\end{array}$ & 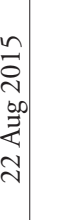 & 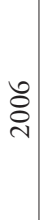 & $\mid \begin{array}{l}0 \\
\text { ¿ } \\
\text { | }\end{array}$ \\
\hline 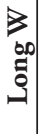 & 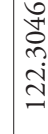 & $\begin{array}{l}\underset{J}{J} \\
\text { N} \\
\stackrel{\text { J }}{J}\end{array}$ & 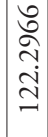 & 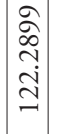 & 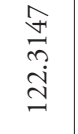 & 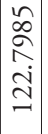 & 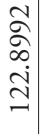 & $\begin{array}{l}\stackrel{+}{ } \\
2 \\
\infty \\
\stackrel{1}{1} \\
\text { I }\end{array}$ & ฮे & 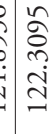 & & 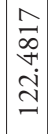 & 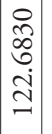 & 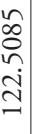 & 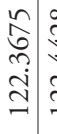 & 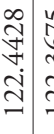 & 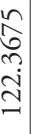 & 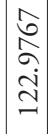 & 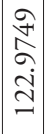 & 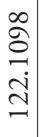 & 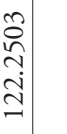 & 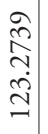 & 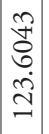 \\
\hline 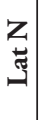 & 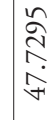 & 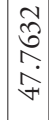 & 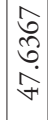 & 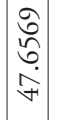 & 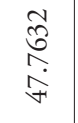 & $\left|\begin{array}{c}\tilde{\alpha} \\
0 \\
\hat{\sigma} \\
\end{array}\right|$ & $\begin{array}{c}\text { ते } \\
\text { ठे } \\
\text { के }\end{array}$ & 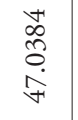 & 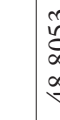 & 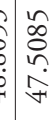 & & $\begin{array}{l}\tilde{n} \\
\hat{\sigma} \\
\alpha \\
\hat{\infty}\end{array}$ & 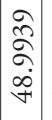 & 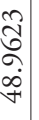 & 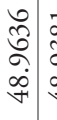 & 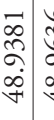 & 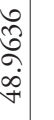 & 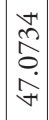 & 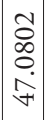 & 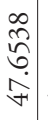 & & 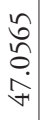 & $\mid \begin{array}{c}c \\
-1 \\
0 \\
o \\
b \\
\forall \\
\forall\end{array}$ \\
\hline ö & है & & 沅 & & $\underset{\square}{\infty}$ & $\left|\begin{array}{c}0 \\
0 \\
0 \\
\vdots \\
\vec{F} \\
\mid\end{array}\right|$ & 8 & $\begin{array}{l}0 \\
\text { D. } \\
\text { 冚 } \\
\text { F }\end{array}$ & 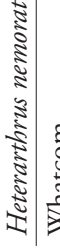 & 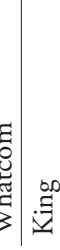 & $\mid$ & 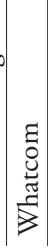 & 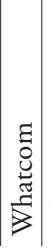 & $\begin{array}{c}\overrightarrow{0} \\
\overrightarrow{0} \\
\overrightarrow{5} \\
\overrightarrow{3}\end{array}$ & 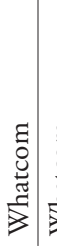 & 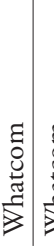 & 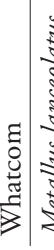 & 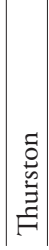 & 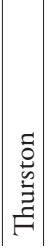 & & 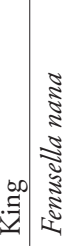 & 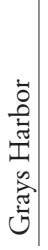 & $\mid$ \\
\hline
\end{tabular}




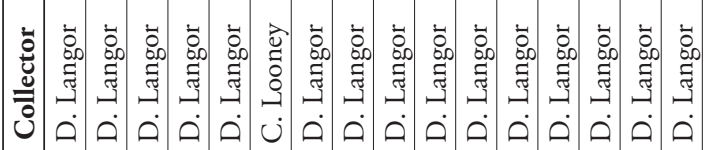

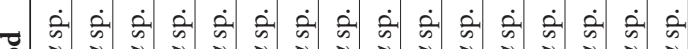

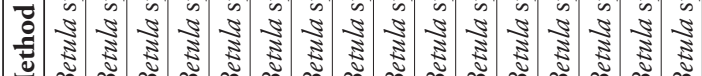

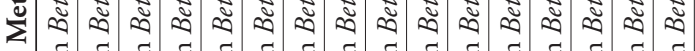
รี

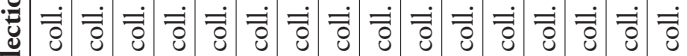

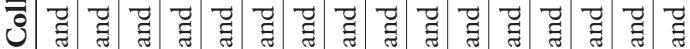

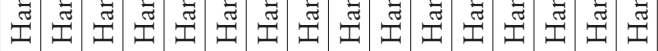

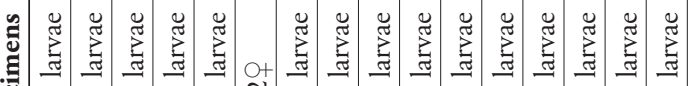

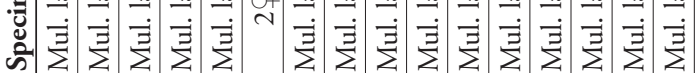

芯

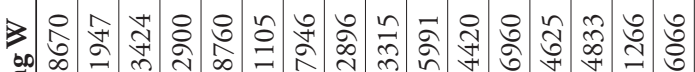
b

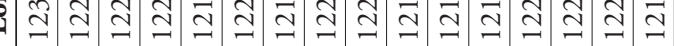

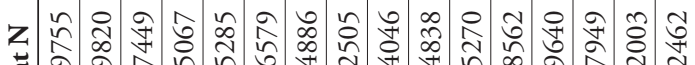
J

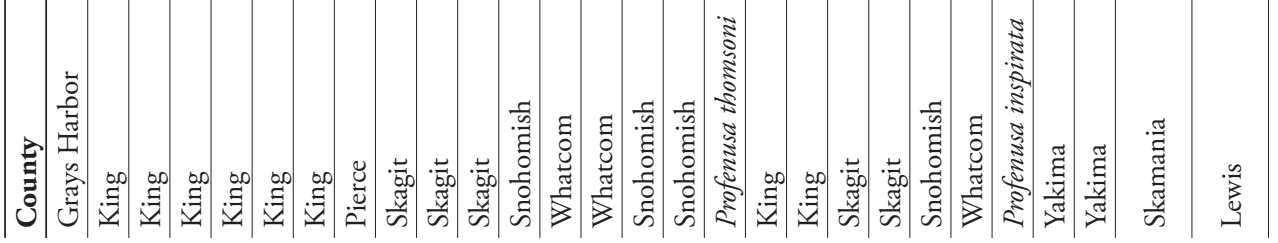

के के के के के

$3: 3 \quad 3$

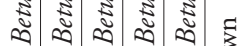

ธี รี

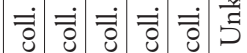

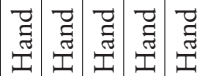

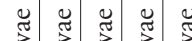

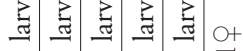

家家家家家
हैं है है है है है है

ह 3 ह 3

竎

‡ 。

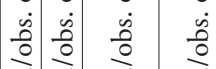

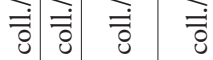

胥

胥

完完家 完

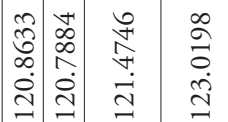

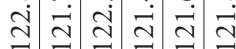

nี 方

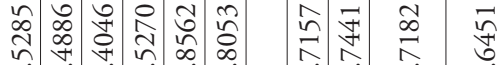

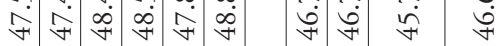




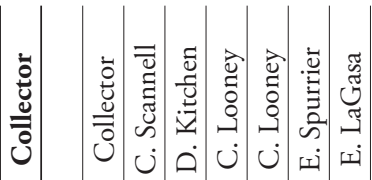

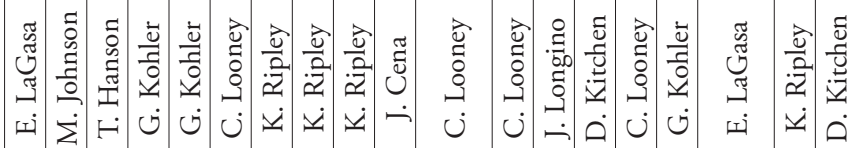

$\frac{\sqrt{3}}{3} \frac{2}{3}$

$\frac{5}{2}$

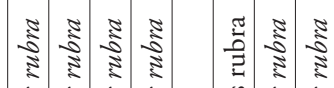

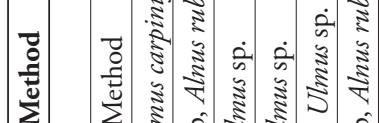

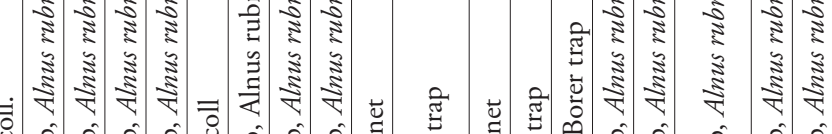

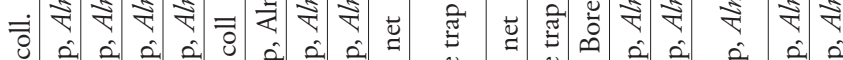

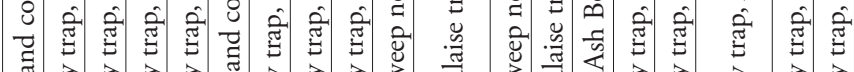

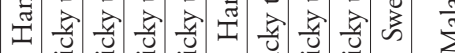

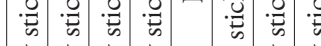

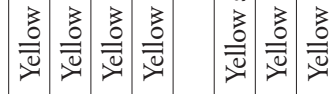

占

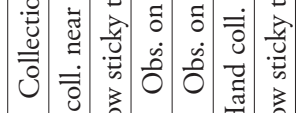

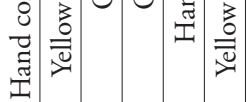

至

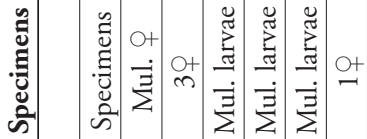

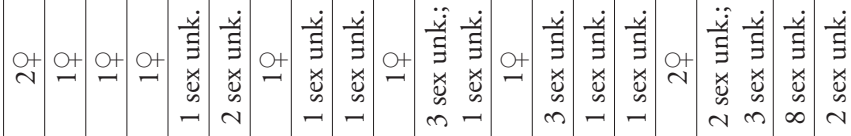

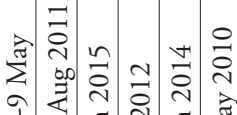

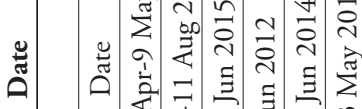

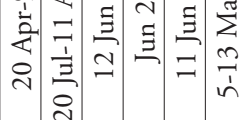

œ

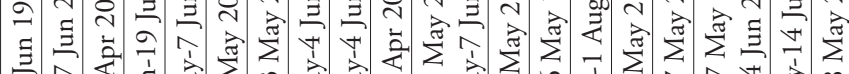

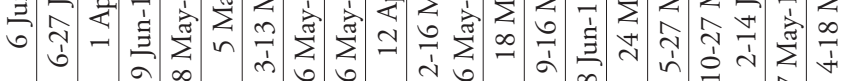

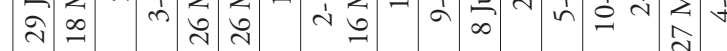

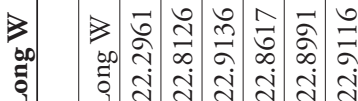

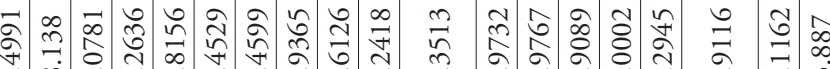

:

ปิ

iे i i

\begin{tabular}{|l|l|l|l|l|}
\hline & \\
\hline
\end{tabular}

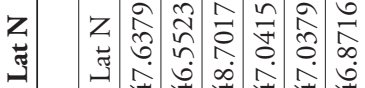

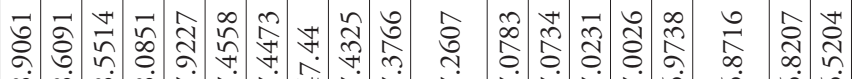

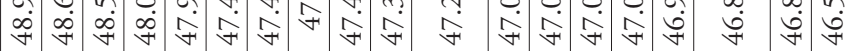

$\frac{2}{2}$

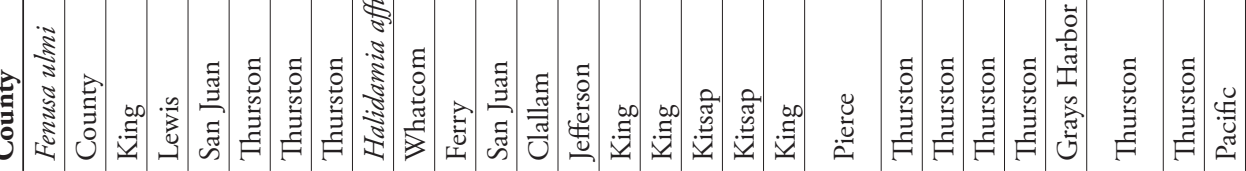




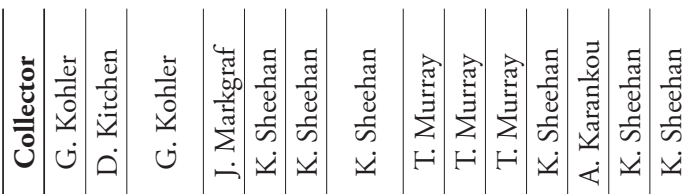

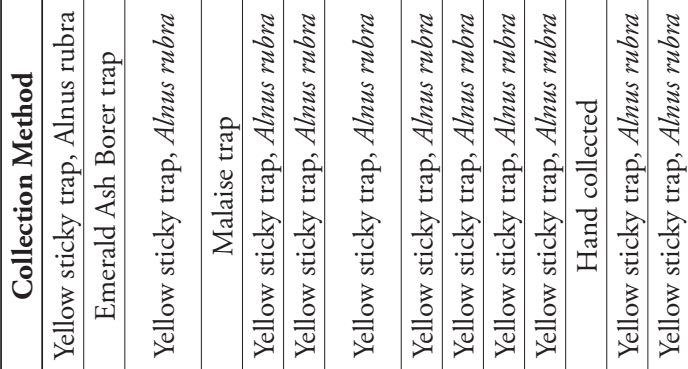

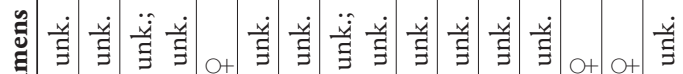

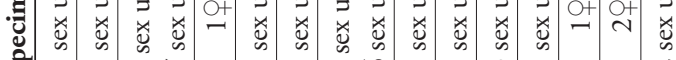
ڤ̆

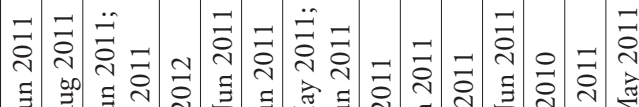

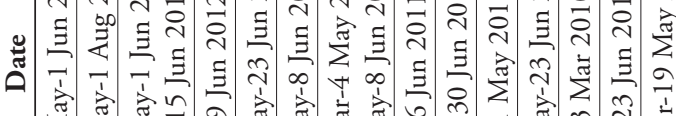

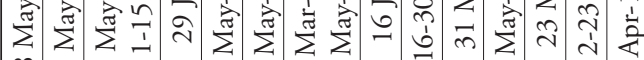

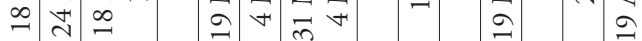

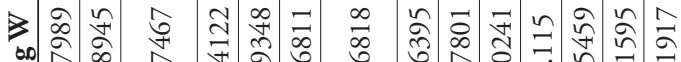

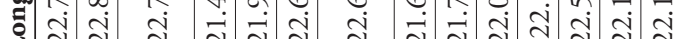

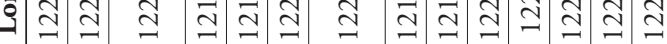

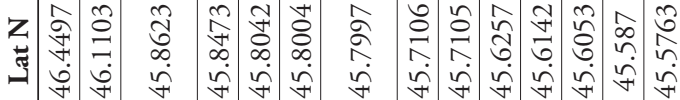

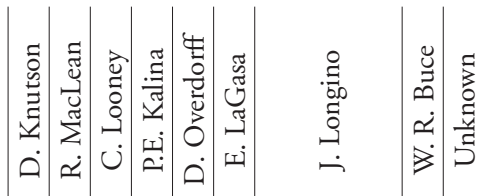

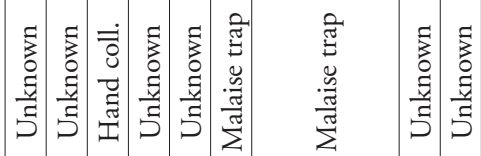

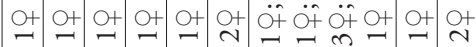

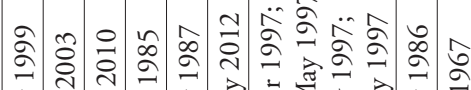

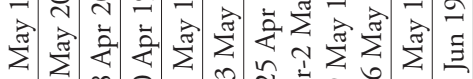

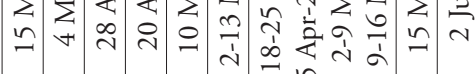

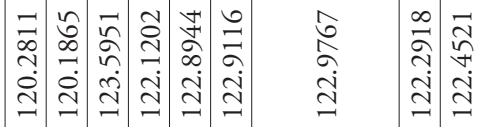

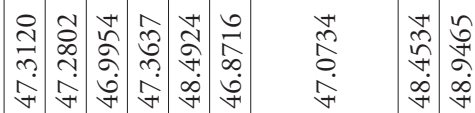

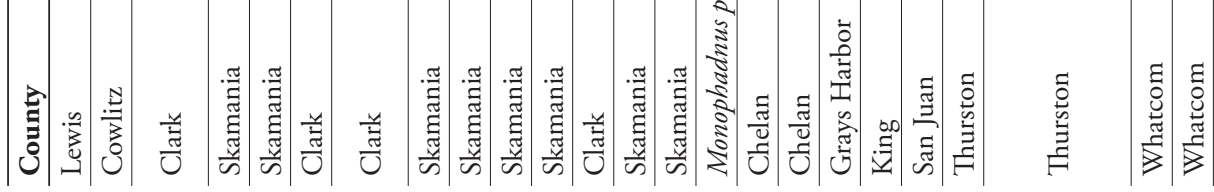




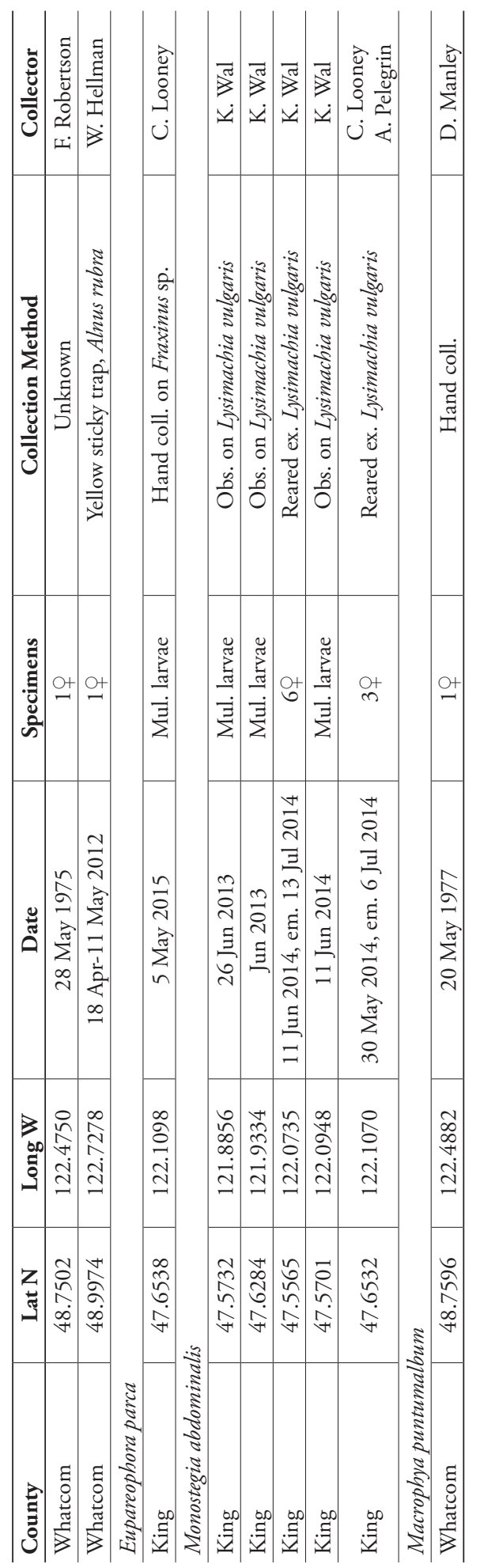




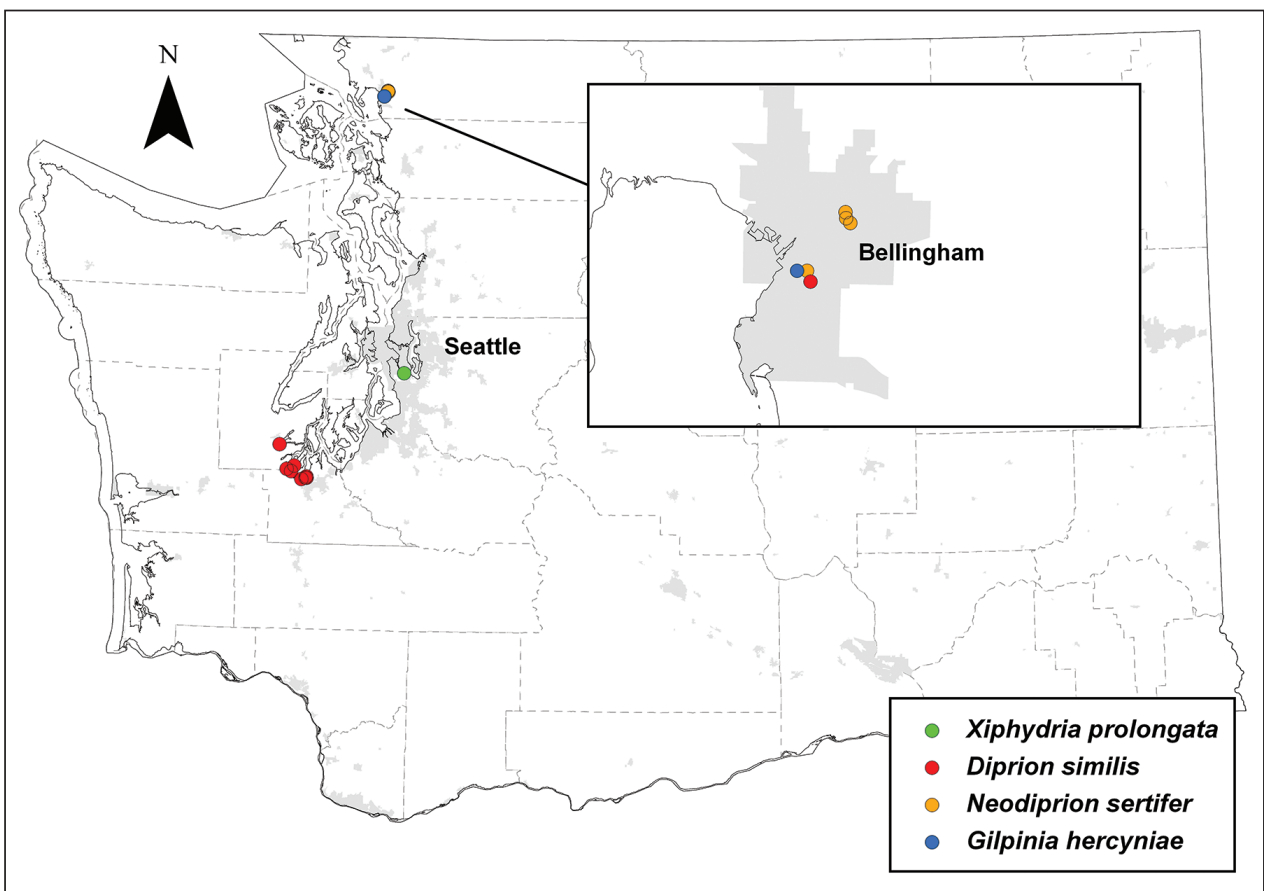

Figure 3. Localities of Xiphydriidae and Diprionidae newly detected in Washington State.

occasionally occur in production forestry, often in association with $D$. pini L., but it is typically not a serious pest (Taeger et al. 1998). The only Diprion recorded from North America (Taeger et al. 2010), D. similis was discovered in New Haven, Connecticut, in 1914, presumably introduced on imported European nursery stock or associated packing materials (Britton 1915). Diprion similis feeds upon multiple pine species, with marked oviposition preference for P. strobus observed in North America (Tsao and Hodson 1956). The species can potentially defoliate entire trees when populations are high; however, this appears to be rare in North America, perhaps due to control by weather events and introduced parasitoids (Wilson 1966, Van Driesche et al. 1996). It is known to occur from the northeastern states westward to the Great Lakes region, and south to North Carolina.

Specimens of D. similis were collected in 2012 when adult females emerged from P. sylvestris boughs collected in Shelton, Washington. Following this detection, yellow card traps were deployed in the south Puget Sound area and Whatcom County. Subsequent visual surveys for larvae were conducted in western Washington. The distinctive larvae are readily recognized, and were found at eight sites in three western Washington counties (Fig. 3). Larvae were most commonly found feeding on P. monticola Douglas ex D. Don, but also on P. sylvestris and P. contorta Douglas ex Loudon. A single male specimen was captured in a Dipion pini pheromone-baited trap, probably by chance 
since the lure is not known to be attractive across species (O. Anderbrant, in litt.). Voucher specimens are deposited at WSDAC and WWUC.

\section{Gilpinia hercyniae (Hartig, 1837)}

Gilpinia comprises 37 described species native to Europe and Asia. Gilpinia hercyniae is a solitary spruce feeder, first detected in Ottawa, Ontario, Canada in 1922 and in New Hampshire in 1929 (Baker 1972). It quickly became a forest pest in the eastern United States and Canada (Balch 1939, Reeks and Barter 1951). Ambitious biological control programs during the 1930s imported and released several parasitoid species throughout the region. Concurrent with this, a nuclear polyhedrosis virus was inadvertently released which resulted in consistent region-wide control (Balch and Bird 1944). The species has not been an active management concern in North America for many decades (Nielon and Morris 1964, Kelleher and Hulme 1984). There are no published records of its occurrence west of Manitoba.

A specimen of $G$. hercyniae was collected in Bellingham in 2008 (although not identified until 2011) from Picea abies (L.) Karst. in a residential neighborhood. Wideranging visual and sticky-trap surveys in northwestern Washington failed to detect it beyond the original site, where more specimens were collected in 2011 and 2012 (Fig. 3). Specimens are housed at WWUC and WSDAC.

\section{Tenthredinidae, Nematinae}

\section{Cladius grandis (Serville, 1823)}

The earliest North American collections for this Palaearctic species are from Albany, New York, in 1887 (Smith 1974a). The species was presumably introduced separately to the west coast, with records from British Columbia in 1914 (Blackmore 1917). The specimens from British Columbia were first described as a new species, Platycampus victoria MacGillivray, 1920, reared from Populus nigra L. (MacGillivray 1920). In most previous literature, the species is known as Trichiocampus viminalis (Fallén, 1808). The most common host plants are Populus spp., although Salix (Benson 1958, Raizenne 1957) and Alnus P. Mill. (Smith 1974a) are also recorded. It has been reported as a minor pest of Populus L. in eastern North America and British Columbia (Béique 1961, Downes 1925). In Quebec, the species is bivoltine, with adults active in late May and early June, and again in late July through September (Béique 1961). There appears to be only one generation per year in British Columbia (Downes 1925). A single specimen from Washington was collected in a Malaise trap on the Evergreen State College campus in 1997 (Fig. 4), and detected while examining unidentified material in the college's natural history museum. The specimen is housed at WSDAC. 


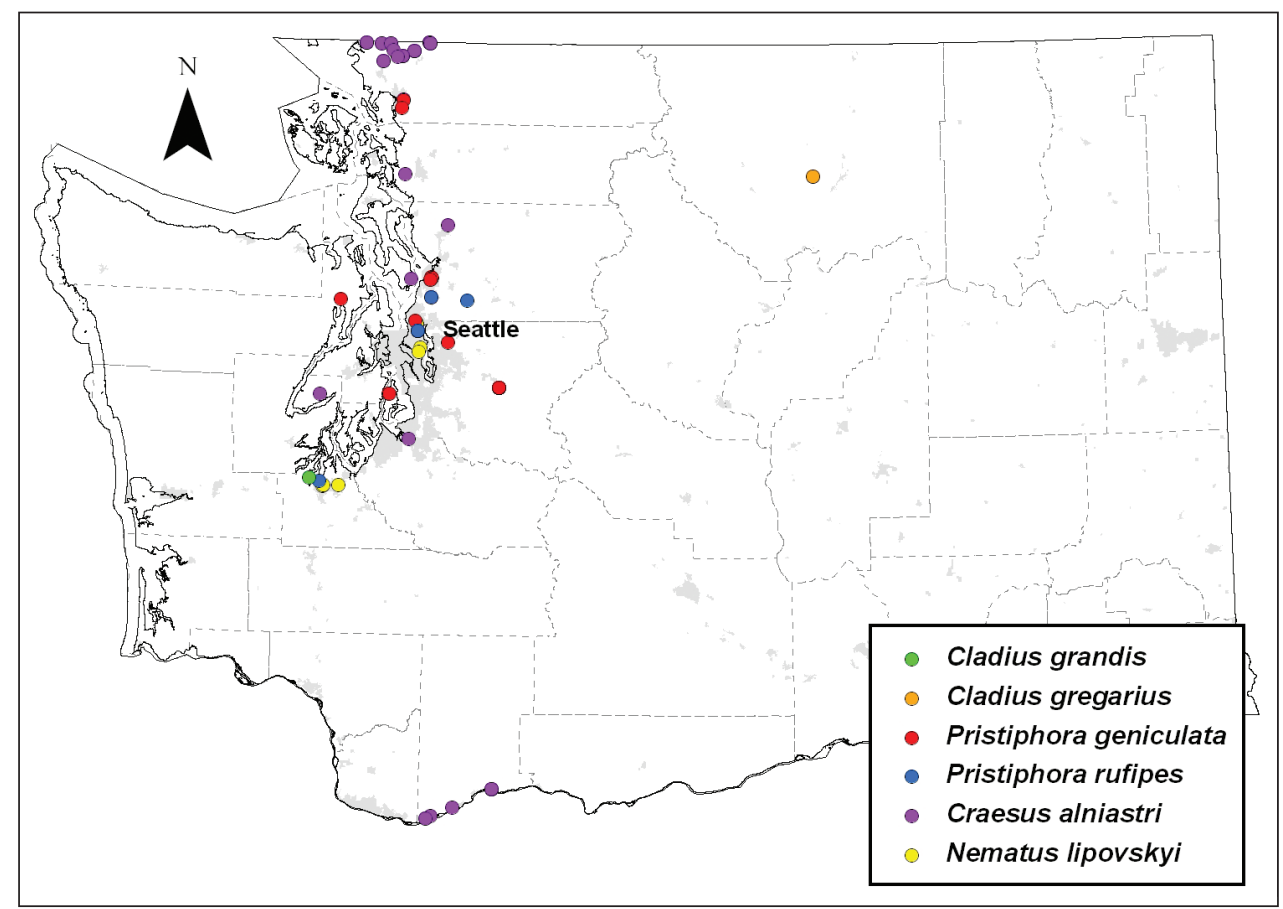

Figure 4. Localities of Nematinae (Tenthredinidae) newly detected in Washington State.

\section{Cladius gregarius Dyar, 1895}

This poplar-feeding species is native to North America, and is known from the northeastern United States and adjacent Canada, west to Michigan and Ontario (Smith 1974a, 1979a). Larvae feed on species of Populus in the spring, and adults can be found in late summer and fall. Two adult specimens were reared from larvae collected on Populus tremuloides Michx. in Okanogan County, Washington, 2010 (Figs 4-5). A visit to the same site in late summer 2013 failed to find more larvae, although similar feeding damage was seen on trees throughout the area. Specimens are housed at WSDAC.

\section{Pristiphora geniculata (Hartig, 1840)}

Pristiphora is a large, primarily Holarctic genus, although there are several described Neotropical and southern Asian species (Taeger et al. 2010). The genus is most diverse in Europe, with ca. 115 known species. Five European Pristiphora spp. introduced to North America are pestiferous, including P. geniculata. This species was first detected in the United States in Haines Falls, New York, and in Massachusetts in 1926, and now occurs throughout the northeastern states and provinces, and west to Minnesota and Ontario (Schaffner 1936, Smith 1979a). Pristiphora geniculata 


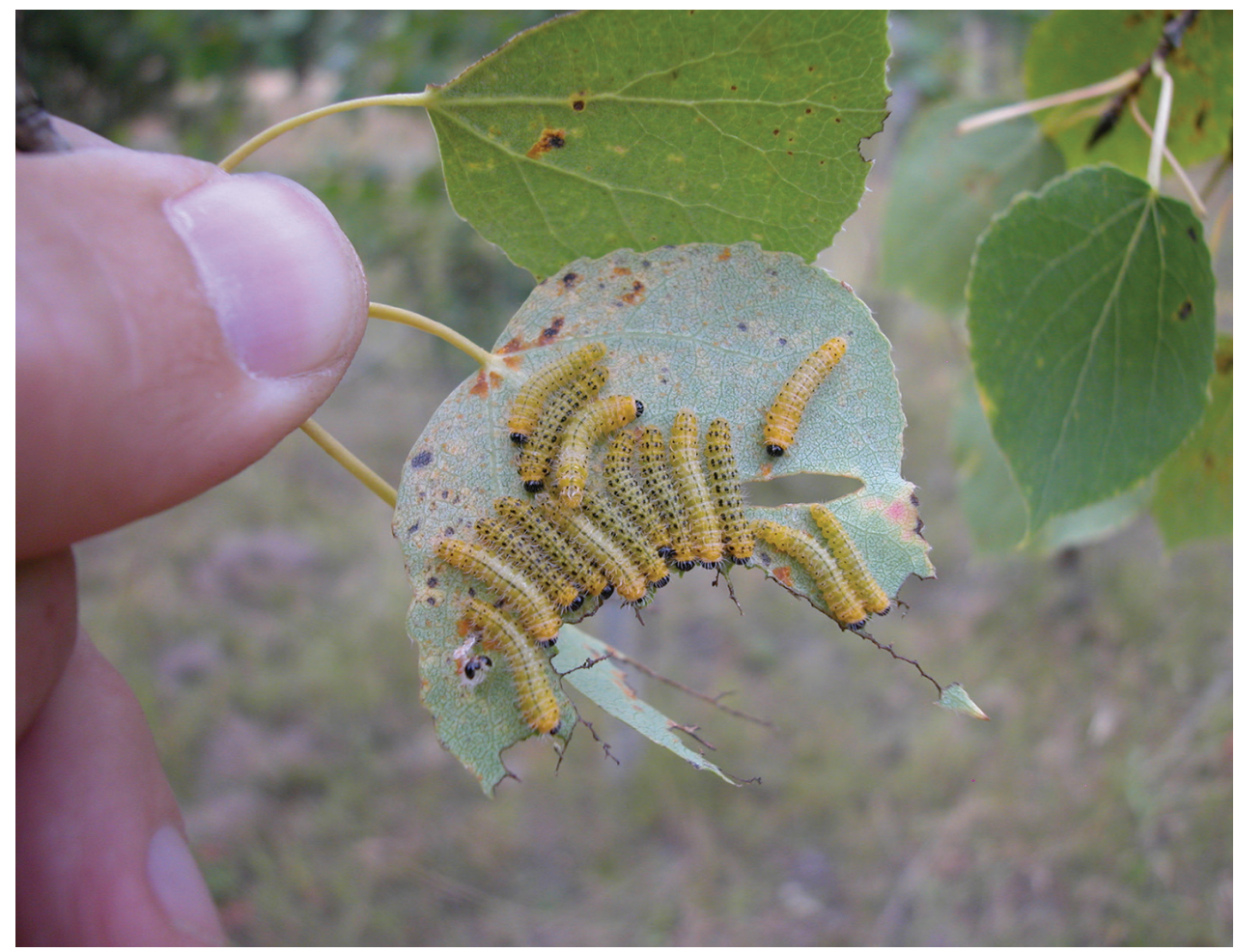

Figure 5. Cladius gregarius larvae on poplar, Okanogan County WA.

feeds on Sorbus aucuparia L., S. americana Marshall, S. decora (Sarg.) C.K. Schneid., and the hybrid cultivar Sorbaronia hybrida (Moench) C.K. Schneid. (Forbes and Daviault 1964). Kunneman and Albers (1991) list P. geniculata as a pest of Tilia L., but this seems to be a misreporting of the "elm sawfly", Cimbex americana Leach, 1817 (Cimbicidae; Dirr 1983, Sinclair et al. 1987). Larvae are voracious feeders and can almost completely defoliate healthy trees; however, mortality of even repeatedly defoliated trees is infrequent (Forbes and Daviult 1964). Release and establishment of the ichneumonid Olesicampe geniculatae Quednau and Lim in the eastern states and provinces has resulted in diminished outbreaks of $P$. geniculata since the 1980s (Kelleher and Hulme 1984, Quednau 1990).

The species was first detected in Washington State in 2009, and is now common throughout the Puget Sound region (Fig. 4). Most specimens have been found via visual survey of defoliated trees, where larvae were readily apparent and subsequently reared. Several adults were reared from Crataegus douglasii Lindl. (a native hawthorn species) in 2011 and 2012, a new host record for the species (Fig. 6). The record in Washington probably represents a separate introduction event, either from its native range or a translocation from eastern North America. Specimens are housed at WSDAC. 


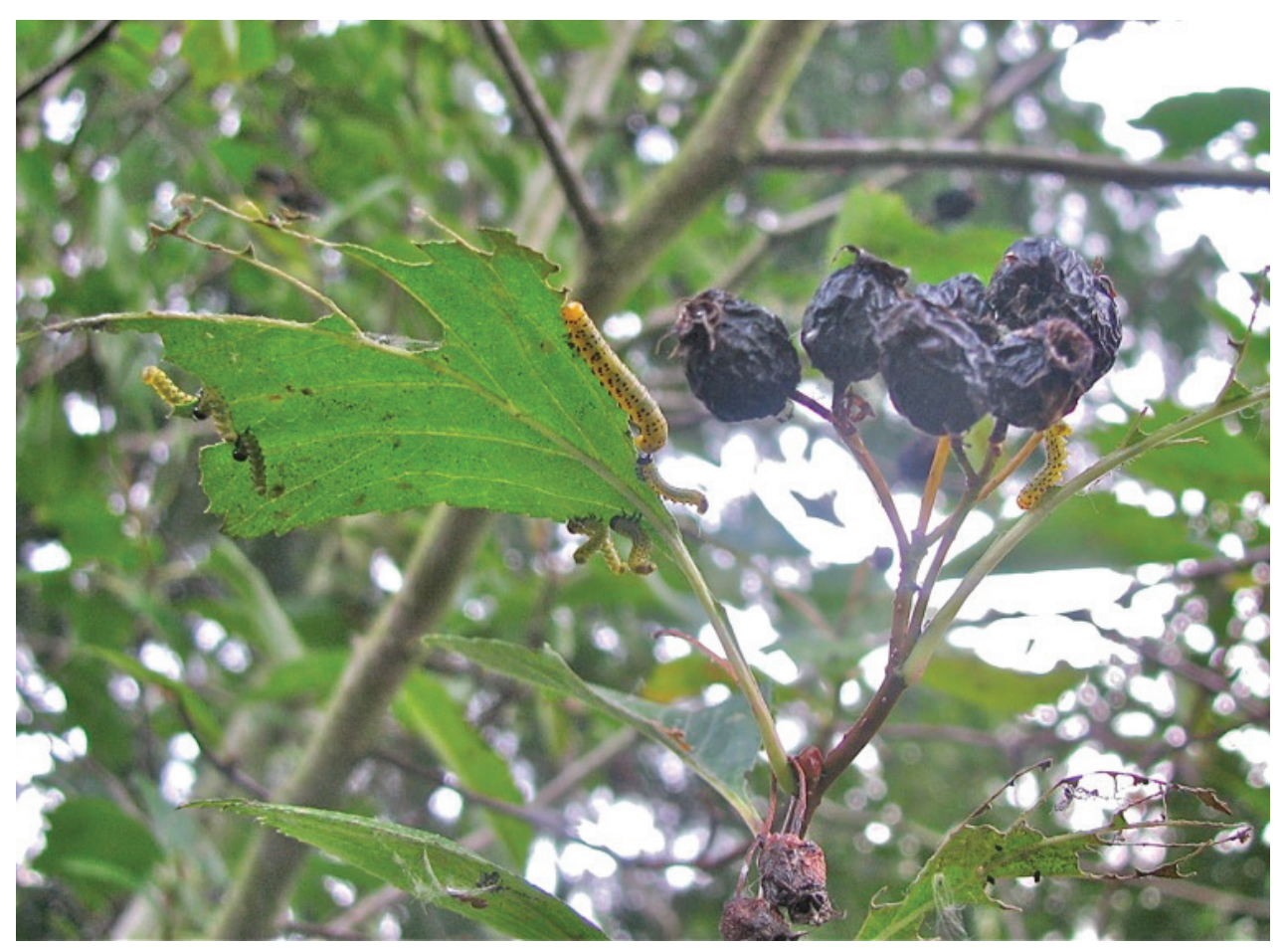

Figure 6. Pristiphora geniculata larvae on Crataegus douglasii, Whatcom County, WA.

\section{Pristiphora rufipes Serville, 1823}

Pristiphora rufipes is native to central Europe, and spread to the United Kingdom in the mid-20 $0^{\text {th }}$ century (Benson 1947). It was first recorded in North America from Ottawa, Canada, in 1963 (MacNay 1964). The species is a significant pest of Aquilegia L. (Alford 2012). The species was previously known from the eastern states and provinces to the midwest. Most North American literature refers to the species by the synonym Pristiphora aquilegiae Snellen van Vollenhoven, 1866. It has been present in Washington since at least 1996, when Seattle-area gardeners began complaining of a new and voracious pest on columbine (Seattle Times 1996). Populations in Washington are currently known from Snohomish, King, and Thurston Counties (Fig. 4). The species has at least three generations per year in western Washington, with larvae present as late as November (Table 1). Specimens are housed at WSDAC.

\section{Craesus alniastri (Scharfenberg, 1805)}

Craesus alniastri is an alder-feeding nematine sawfly native to Europe, where it has been well-studied for its distinctive larval feeding aggregations (e.g., Boevé 1991). Buckle (1930) collected a single specimen of $C$. alniastri on parsnip flowers near Mt. Royal 
(Montreal), Quebec, Canada, in 1926, and Kirby (1882) lists a single male specimen housed in the Natural History Museum, London, that was collected in Nova Scotia. Smith (1972) does not report any further specimens, and there appears to be no further literature discussing the species in North America. Prous et al. (2014) reassign species in this genus to Nematus.

Numerous specimens of $C$. alniastri were collected in western Washington while conducting surveys for alder-feeding sawflies throughout the Pacific Northwest in 2009-2011 (Fig. 4). Adult specimens were collected from May through August. Specimens were collected very near the British Columbia and Oregon borders, indicating that the species is likely widespread in the region. An additional female specimen collected near Seattle in 1976 is in the USNM; other voucher specimens are deposited at WSDAC.

\section{Nematus lipovskyi Smith, 1974}

This species was described from the eastern United States (Smith 1974b) and is known from Maine to Alabama, and west to Wisconsin. It is recently established in the Czech Republic (Macek and Śípek 2014). Larvae feed on deciduous azaleas in the Pentanthera subgenus and section of Rhododendron (e.g., R. viscosum (L.) Torr., R. molle (Blume) G. Don, $R$. luteum Sweet, and $R$. calendulaceum (Michx.) Torr.). Macek and Šípek (2014) also noted feeding damage on $R$. obtusum (Lindl.) Planch. inflorescences, but proposed that this was opportunistic feeding due to the proximity of more suitable host plants. This species is reassigned to Euura in Prous et al. (2014).

A 1996 specimen from Lacey, Washington, is the earliest record from the western USA. Larvae have been observed in several locations in King, Thurston, and Clark counties (Fig. 4). Adults were collected in Olympia, Washington, in April 2015, and are stored at WSDAC.

\section{Tenthredinidae, Heterarthrinae}

\section{Heterarthrus nemoratus (Fallén, 1808)}

Heterarthrus is a relatively small genus of leaf-mining sawflies native to Europe and Asia, which generally mine leaves of trees in Aceraceae, Betulaceae, and Salicaceae (Taeger et al. 2010). Two species have been introduced to North America, H. nemoratus and $H$. vagans (Fallén, 1808), which mine species of Betula L. and Alnus, respectively (Smith 1971, Taeger et al. 1998, Humble 2010). Heterarthrus nemoratus was first collected in North America in Pictou, Nova Scotia, in 1905 (Dowden 1941), and now has a transcontinental distribution in Canada (Digweed et al. 2009). Digweed et al. (2009) review the biology and provide detailed keys to mines, larvae, and adults of this and other birch-feeding Heterarthrinae in North America. The earliest known Wash- 


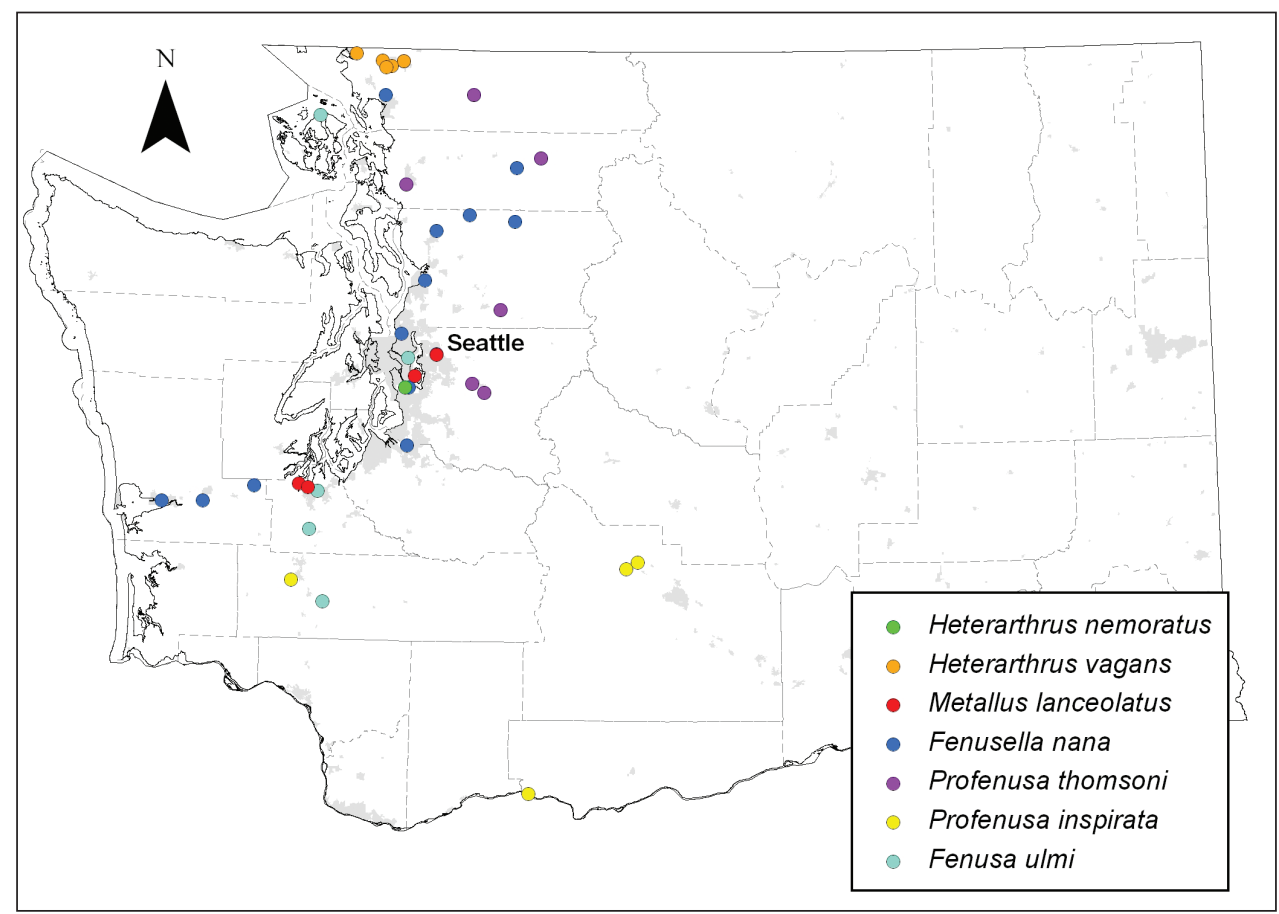

Figure 7. Localities of Heterarthrinae (Tenthredinidae) newly detected in Washington State.

ington specimen is a female collected in Whatcom County (the northern-most county in western Washington) in 1967, housed at WWUC. Specimens are also known from the Seattle area (Fig. 7), and the species is likely widespread in western Washington.

\section{Heterarthrus vagans (Fallén, 1808)}

Heterarthrus vagans was recently detected in North America, discovered in British Columbia in 2009 in numerous locations west of the Cascade Range and very close to the Washington State border (Humble 2010). Visual and sticky trap surveys in 2011 and 2012 detected the species at six sites in Whatcom County (Fig. 7). We have not yet found the species south of Whatcom County, but the widespread distribution of alder makes it likely that this species will continue to spread through Washington and into Oregon. This species' mines are identifiable by the round cocoon formed in the leaves of its host plants; other Heterarthrus spp. pupate outside of leaves (Humble 2010). Specimens are housed at WSDAC.

\section{Metallus lanceolatus (Thomson, 1870)}

Metallus lanceolatus (referenced as M. gei (Brishcke, 1883) in much of the European literature) is a leaf-miner of Geum L. The earliest North American specimens of this Palaearctic species were collected in British Columbia in 1933, and multiple specimens were collected 
in the northeastern USA and Canada in the 1960s (Smith 1971). Smith (1971) described $M$. bensoni as a new species from the New York and British Columbia specimens; Koch (1989) subsequently recognized $M$. bensoni and $M$. gei as junior synonyms of the Palaearctic $M$. lanceolatus. Metallus lanceolatus forms coalescing blotch-mines in ornamental and wild Geum, and can be a pest of garden plants (Buhr 1941, Hoebeke and Wheeler 2005). It has apparently spread through southern Puget Sound (Fig. 7), where it was found attacking Geum macrophyllum Willd. in cultivated and wild conditions. Specimens have been handcollected and captured in Malaise traps. The earliest mines in a large patch of G. macrophyllum near Olympia, Washington, were visible by early July in 2012 and 2013, and could be found on nearly every plant by mid-July of both years. Specimens are housed at WSDAC.

\section{Fenusella nana (Klug, 1816)}

This Palaearctic species is commonly recorded in the literature as Messa nana (see Taeger and Blank 1998 for nomenclatural discussion). Fenusella nana larvae form coalescing blotch mines in many birch species (e.g., Buhr 1941, Taeger et al. 1998, Digweed et al. 2009). The first North American records for this leafminer are from Maine in 1966 (Smith 1967). It has since spread across Canada and into Washington State (Digweed et al. 2009). Digweed et al. (2009) review its biology and provide detailed keys to mines, larvae, and adults of this and other birch-feeding Heterarthrinae in North America. This species has been collected as far southwest as Hoquiam, Grays Harbor County (Fig. 7). Voucher specimens are housed at the NRFC.

\section{Profenusa thomsoni (Konow, 1886)}

The first North American records for this birch leafminer are from Hamden, Connecticut in 1926. It was known from Maine, Ontario, Quebec, and Vermont by the 1960s (Smith 1971), and has since spread across Canada and into Alaska, the Yukon Territory, and Northwest Territories (Digweed and Langor 2004, Digweed et al. 2009, MacQuarrie et al. 2013). It is a significant pest in urban forests (Martin 1960, Drouin and Wong 1984, Snyder et al. 2007). Digweed et al. (2009) review its biology and provide detailed keys to mines, larvae, and adults of this and other birch-feeding Heterarthrinae in North America. The earliest Washington specimen was collected in 1967, in Whatcom County, and was discovered by examining specimens housed at WWUC. Numerous specimens have also been collected south to King County (Fig. 7) and are housed at NRFC.

\section{Profenusa inspirata (MacGillivray, 1909)}

Profenusa inspirata, a native North American species, is the only known sawfly leafminer of oaks in western North America. It creates blotch mines in the upper surface of oak leaves (Fig. 8), that can coalesce when multiple larvae are present in a leaf. The 


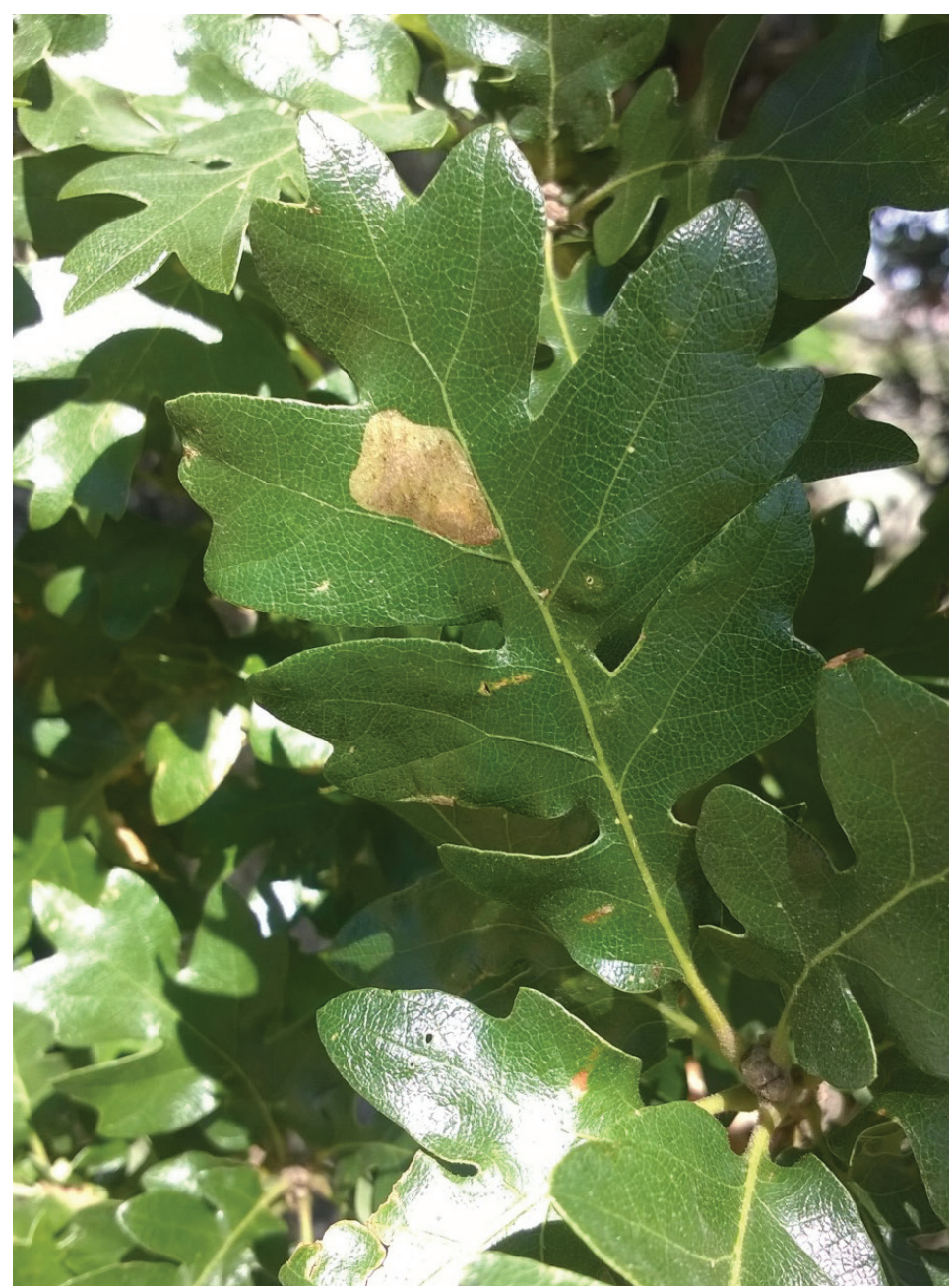

Figure 8. Profenusa inspirata mines on Quercus garryana, Yakima County, Washington.

species was previously known from Nevada, California, and Oregon (Smith 1971). Multiple mines were observed in Garry oak (Quercus garryana Douglas ex Hook.) along the Tieton River, Yakima County, and in Skamania and Lewis counties in 2015 (Fig. 7). Larvae were identified using characters described in Smith (1971). Voucher specimens are at WSDAC.

\section{Fenusa ulmi Sundevall, 1847}

This Palaearctic elm leafminer was already well established in New York by 1898 (Felt 1898), and was certainly introduced with elms from Europe. It is known to attack several elm species, especially Ulmus glabra Huds. and its hybrids (Slingerland 1905, Liston 1993, Scannell 2000); Scannell (2000) added U. pumilla L. and U. davidiana 


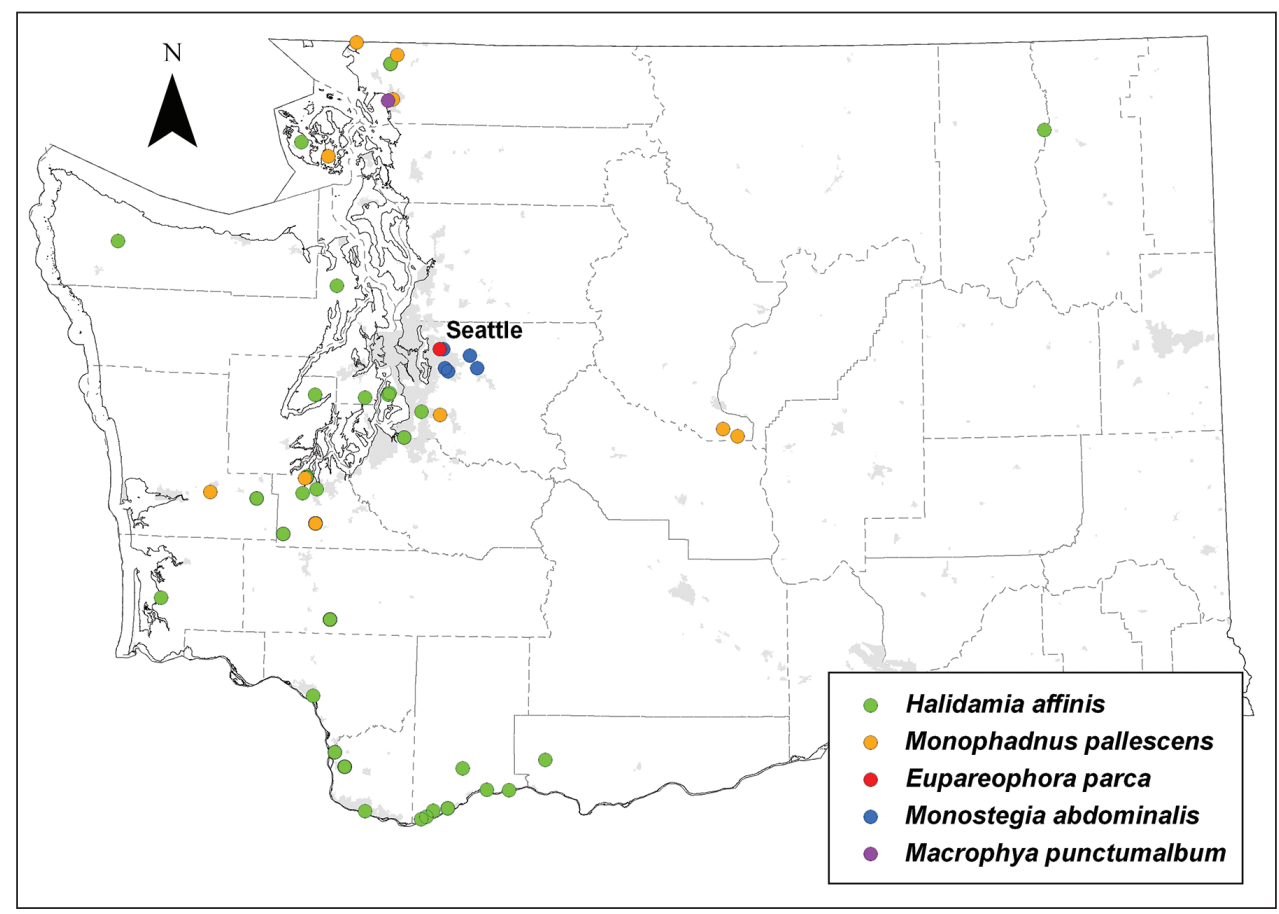

Figure 9. Localities of Blennocampinae, Allantinae, and Tenthredininae (Tenthredinidae) newly detected in Washington State.

Planch. to the known elm hosts. Early records of significant damage to $U$. americana L. seem to be unsubstantiated, with most authors reporting that larvae are unable to do more than initiate feeding (Slingerland 1905, Guries and Smalley 1994, Scannell 2000). Scannell (2000) provided detailed information on the life history and host preferences of this species in the Seattle area. The oldest known west coast specimens were collected in British Columbia in 1947, and are stored in the Canadian National Collection (H. Goulet, pers. comm.). There is anecdotal evidence that the species has been present in Washington since the early 1990s (Scannell 2000), and has since spread south through the Puget Sound area (Fig. 7). Washington State voucher specimens are at WSDAC.

\section{Tenthredinidae, Blennocampinae}

\section{Halidamia affinis (Fallén, 1807)}

This is an introduced European species that feeds on Galium L (Smith 1969, Taeger et al. 1998). The earliest North America records are 1931 from Cold Spring Harbor, New York, 1933 from Connecticut, and 1934 from New Jersey (Smith 1969). It has since spread to California and the Pacific Northwest. The species is common and was col- 
lected as larvae from host plants and readily on yellow sticky traps and in Malaise traps in nearly every county surveyed (Fig. 9). Voucher specimens are at WSDAC.

\section{Monophadnus pallescens (Gmelin, 1790)}

A Ranunculus-feeding European introduction, this species has been present in the eastern United States and Canada since the late 1800s and in British Columbia since at least 1919 (Smith 1969). This species is widespread (Fig. 9), and was collected with sticky card traps, Malaise traps, and by hand. The earliest specimens discovered from Washington were collected in Whatcom County in the late 1960s, and were found in the material at WWUC. Numerous species of Ranunculus L. provide suitable hosts in western Washington, including the widespread invasive European weed Ranunculus repens L. Voucher specimens are housed at WWUC and WSDAC.

\section{Eupareophora parca (Cresson, 1880)}

The genus Eupareophora contains three species, with only E. parca native to North America. It is known from most of the northeastern and central states, and north and west to Alberta (Williams 2007). The species is not known from the Rocky Mountains, but has been collected from several northern California locations and Oregon (Smith 1969). The single historical specimen from Oregon was collected by Koebele, who collected in and around California in the 1880s. Most collection records for this western disjunct population are from California (Smith 1969), suggesting the species may have been native to eastern North America and spread west via human commerce. Williams (2007) similarly suggests that the species was not present in Alberta until the early 2000s. Several Fraxinus L. species are recorded hosts, as well as Carya illinoinensis (Wangenh.) K. Koch (Smith 1969) and Chionanthus L. (Dyar 1898), although Smith (1969) described the latter association as "dubious". Larvae are readily recognizable by the bristly appearance caused by numerous thick, bifurcate spines. Williams (2007) presented a very detailed account of larval and adult biology in Alberta. Heavy ash defoliation and shed skins of an unknown sawfly were noticed at a public park on the border of King and Snohomish counties in 2014, but no living animals were observed. A return visit in May 2015 found the easily identifiable larvae feeding on Fraxinus latifolia Benth. throughout the park (Fig. 9). Voucher specimens are at WSDAC.

\section{Tenthredinidae, Allantinae}

\section{Monostegia abdominalis (Fabricius, 1798)}

Monostegia abdominalis is a European sawfly pestiferous on Glaux L., Lysimachia L. and Anagallis L. (Price 1970, Taeger et al. 1998). It was first collected in North America 
in Massachusetts in 1899, and described as M. martini MacGillivray, 1908 (Smith 1979b). In late summer of 2013, noxious weed management staff in King County noticed heavy defoliation of populations of Lysimachia vulgaris L., regulated as a noxious weed in Washington State, by unknown sawfly larvae. Larvae were subsequently collected and reared in the lab on a Lysimachia cultivar in 2014. Specimens are known from several Lysimachia infestations in western Washington (Fig. 9). Voucher specimens are at WSDAC.

\section{Tenthredinidae, Tenthredininae}

\section{Macrophya punctumalbum (Linnaeus, 1767)}

This adventive European species was first recorded in North America from Toronto, Ontario, in 1932, from British Columbia in 1934 (Gibson 1980), and later from New York (Hoebeke and Johnson 1985). Larvae feed on Ligustrum L., Syringa L., and Fraxinus (Gibson 1980). A specimen collected in 1977 in Bellingham, Washington, and housed at WWUC is the only record from Washington State (Fig. 9).

\section{Discussion}

In addition to expanding regional knowledge of an ecologically interesting and economically important group of insects, these data highlight the continual intra-continental spread of introduced species. Eighteen of the 22 sawflies reported here represent range expansions for exotic sawflies introduced to North America long ago. Most of these species were first recorded from eastern states or provinces, likely introduced with nursery stock. Five species may have been first or simultaneously introduced on the west coast based on their historical detection data - Cladius grandis, Heterarthrus vagans, Metallus lanceolatus, Monophadnus pallescens, and Macrophya punctumalbum. It is unknown how most of the introduced species became established in the west. Certainly, commerce from eastern North America could explain the distribution of some species. For some pests of common cultivated plants, such as Pristiphora rufipes and Neodiprion sertifer, it seems probable that insects were transported inadvertently with nursery trade or by home gardeners, although natural dispersal by adults can not be discounted.

The movement of other species, such as Halidamia affinis and Monostegia abdominalis, is more mysterious - Galium is not commonly cultivated, and Lysimachia vulgaris is a noxious weed. Halidamia affinis has likely spread of its own accord, expanding through the immense range of its host plant, Galium. Monostegia may have moved with other cultivars of Lysimachia that are commercially sold, although one would expect that such voracious and notable sawfly larvae on garden plants would have been reported. The reports of previously more southern species, such as Profenusa inspirata and Eupareophora parca could indicate northward range expansion concurrent with in- 
creasingly moderate winters. Profenusa inspirata in particular seems suggestive of such new expansion, since Garry oak conservation and ecology have long been studied in the Puget Sound region. However, it is certainly possible that P. inspirata has been present but undetected in Washington for decades.

The older specimens recorded here from collections made decades ago emphasize the value of institutional insect collections as repositories of valuable biodiversity information. It is noteworthy that for several of the species discussed herein, the first records for the state were found in the holdings of the insect collections at regional universities, providing evidence that many species had been transported west earlier than was previously known, or were perhaps derived from separate introduction events. Thus, despite the relatively small size of such collections compared to those at land grant universities, these collections fill a valuable role in documenting shifts in regional species composition. As a whole, the data from all museum specimens examined during this research also demonstrate gaps in our regional knowledge of sawflies. Unsurprisingly given remoteness and lack of access, the arid Columbia Basin and rugged mountain ranges in Washington are less frequently collected than other parts of the state (Fig. 2). Undoubtedly, other sawfly species occur in Washington that as yet are undetected and perhaps limited to these under-surveyed habitats. For example, the juniper-feeding genus Susana Rohwer \& Middleton, 1932 occurs in states and provinces surrounding Washington but has not been recorded from the state.

\section{Acknowledgements}

We thank Kaile Adney, Jennifer Andreas, Rachel Chai, Caitlin Fate, Maggie M. Freeman, Thor Hanson, Warren E. Hellman, Amelia Jordan, Don Kitchen, Glenn Kohler, Andi Kopit, Eric LaGasa, Diane Maclean, Joy Markgraf, Todd Murray, Arlo Pelegrin, Karen Ripley, Alexis Sarah, Kathy Sheehan, Holli Watne, and Sue Welch for field and/ or lab assistance. Tim Cahill and multiple anonymous property owners generously allowed access to collecting sites. Frank Merickel (University of Idaho), Chris Marshall (Oregon State University), Jack Longino and Brendan Boudinot (The Evergreen State College), and Richard S. Zack (Washington State University) graciously facilitated research at their respective institutional entomology collections. We thank two anonymous reviewers for comments and observations that significantly improved the readability and professionalism of this article. The data in this report were collected in part with funding from the USFS Forest Health Monitoring Program and USDA-APHIS Cooperative Agreements \#11-8550-1505-CA and \#11-8550-1502-CA.

\section{References}

Alford DV (2012) Pests of Ornamental Trees, Shrubs and Flowers. Manson Publishing, London, 480 pp. doi: $10.1201 / \mathrm{b} 15136$ 
Baker WL (1972) Eastern forest insects. US Department of Agriculture Miscellaneous publication 1175. Washington, DC.

Balch RE (1939) The outbreak of the European spruce sawfly in Canada and some important features of its bionomics. Journal of Economic Entomology 32: 412-418. doi: 10.1093/ jee/32.3.412

Balch RE, Bird FT (1944) A disease of the European spruce sawfly, Gilpinia hercyniae (Htg.), and its place in natural control. Scientific Agriculture 25: 65-80.

Béique R (1961) Étude sur la mouche-à-scie du peuplier, Trichiocampus viminalis (Fall.) (Hyménoptère: Tenthredinidae). The Canadian Entomologist 93: 1085-1097. doi: 10.4039/ Ent931085-12

Benjamin DM, Larson JD, Drooz AT (1955) The European pine sawfly on the Henderson State Forest, Illinois, with notes on its biology and control. Journal of Forestry 53: 359-62 Benson RB (1947) The columbine sawfly (Pristiphora alnivora Hartig) (Hym., Tenthredinidae) in Britain, in Middlesex and Devon. Entomologists Monthly Magazine 83: 223.

Benson RB (1958) Hymenoptera: 2. Symphyta. Section (c). Handbooks for the Identification of British insects. Vol. VI, Part 2(c), Royal Entomological Society of London, 139-252.

Blackmore EH (1917) Entomology. Report of the Provincial Museum of Natural History for the Year 1917. Government of British Columbia, Victoria.

Boevé J-L (1991) Gregariousness, field distribution and defence in the sawfly larvae Croesus varus and C. septentrionalis (Hymenoptera, Tenthredinidae). Oecologia 85: 440-446. doi: 10.1007/BF00320623

Britton WE (1915) A destructive pine sawfly introduced from Europe, Diprion (Lophyrus) simile Hartig. Journal of Economic Entomology 8: 379-382. doi: 10.1093/jee/8.3.379

Buckle JW (1930) Croesus varus (de Vilaret) (Hymenoptera). The Canadian Entomologist 62: 21-22. doi: 10.4039/Ent6221-1

Buhr H (1941) Beobachtungen über Nahrungspflanzen, Verbreitung und Auftreten von minierenden Blattwespen. Mitteilungen der Münchner Entomologischen Gesellschaft 31: 903-926.

Canadian Forest Service (2005) Alien forest pests: context for the Canadian Forest Service's Science Program. CFC Science Branch, Ottawa, Ontario, Canada.

Cassidy KM, Grue CE, Smith MR, Dvornich KM (1997) Washington State Gap Analysis Final Report. Volumes 1-5. Washington Cooperative Fish and Wildlife Research Unit, University of Washington, Seattle, USA.

Craighead FC (1950) Insect Enemies of Eastern Forests. US Department of Agriculture, Miscellaneous Publication 657, 679 pp.

Day KR, Leather SR (1997) Threats to forestry by insect pests in Europe. In: Watt AD, Stork NE, Hunter MD (Eds) Forests and Insects. Chapman and Hall, London, 177-205.

Deyrup MA (1984) A maple wood wasp, Xiphydria maculata, and its insect enemies. The Great Lakes Entomologist 17: 17-28.

Digweed SC, Langor DW (2004) Distributions of leafmining sawflies (Hymenoptera: Tenthredinidae) on birch and alder in northwestern Canada. Canadian Entomologist 136: 727-731. doi: 10.4039/n03-096 
Digweed SC, MacQuarrie CJK, Langor DW, Williams JM, Spense JR, Nystrom KL, Morneau L (2009) Current status of invasive alien birch-leafmining sawflies (Hymenoptera: Tenthredinidae) in Canada, with keys to species. Canadian Entomologist 141: 210-235. doi: 10.4039/n09-003

Dirr MA (1983) Manual of Woody Landscape Plants: Their Identification, Ornamental Characteristics, Culture Propagation and uses. Stipes, Champaign, 759 pp.

Dowden PB (1941) Parasites of the birch leaf-mining sawfly (Phyllotoma nemorata). U. S. Department of Agriculture Technical Bulletin 757. Washington, D.C.

Downes W (1925) The poplar sawfly (Trichiocampus viminalis (Fallén)). Proceedings of the Entomological Society of British Columbia 22: 26-32.

Drouin JA, Wong HR (1984) Birch leaf-mining sawflies in Alberta (Hymenoptera: Tenthredinidae). Information Report NOR-X-260, Canadian Forest Service, Environment Canada, Edmonton, Alberta.

Felt EP (1898) Notes on some of the insects of the year in the state of New York. United States Department of Agriculture Division of Entomology, Bulletin 17.

Forbes RS, Daviault L (1964) The biology of the mountain-ash sawfly Pristiphora geniculata (Htg.) (Hymenoptera: Tenthredinidae), in eastern Canada. The Canadian Entomologist 96: 1117-1133. doi: 10.4039/Ent961117-8

Gibson GAP (1980) A revision of the genus Macrophya Dahlbom (Hymenoptera: Symphyta, Tenthredinidae) of North America. Memoirs of the Entomological Society of Canada 114: $1-167$.

Goulet H (1986) The genera and species of the Nearctic Dolerini (Symphyta: Tenthredinidae: Selandriinae): classification and phylogeny. Memoirs of the Entomological Society of Canada 135: 1-208. doi: 10.4039/entm118135fv

Goulet H (1996) Revision of the Nearctic species of the Arcuata group of the genus Tenthredo with notes on the higher classification of the Tenthredinini (Hymenoptera, Symphyta, Tenthredinidae). Contribution of the American Entomological Institute 29: 1-135.

Griffiths KJ (1959) Observations on the European pine sawfly, Neodiprion sertifer (Geoff.), and its parasites in southern Ontario. The Canadian Entomologist 91: 501-512. doi: 10.4039/ Ent91501-8

Gurries RP, Smalley EB (1994) Variation in elm leafminer injury on elms in Wisconsin. Proceedings of the Eighth METRIA Conference. METRIA and the International Ornamental Crabapple Society. U.S. National Arboretum, Washington DC.

Humble LM (2010) First North American records for Heterarthrus vagans (Hymenoptera: Tenthredinidae), a Palaearctic leafmining sawfly of alder. The Canadian Entomologist 142: 181-187. doi: 10.4039/n09-071

Hoebeke ER, Johnson WT (1985) A European privet sawfly, Macrophya punctumalbum (L.): North American distribution, host plants, seasonal history and descriptions of the immature states (Hymenoptera: Tenthredinidae). Proceedings of the Entomological Society of Washington 87: 25-33.

Hoebeke ER, Wheeler AG Jr (2005) First records of adventive Hymenoptera (Argidae, Megachilidae, Tenthredinidae, and Vespidae) from the Canadian Maritimes and the United States. Entomological News 116: 159-166. 
Kelleher JS, Hulme MA (1984) Biological Control Programmes against Insects and Weeds in Canada. 1969-1980. CAB, Slough.

Kirby WF (1882) List of the Hymenoptera, with descriptions and figures of the typical specimens in the British Museum Vol 1. Tenthredinidae and Siricidae. London, 478 pp.

Kunneman BPAM, Albers MRJ (1991) Linden Trees (Tilia spp.). In: Bajaj YPS (Ed.) Trees III, Biotecnhology in Agriculture and Forestry V. 16. Springer-Verlag, Berlin, 152-163. doi: 10.1007/978-3-662-13231-9_9

Kruse JJ, Smith DR, Schiff NM (2010) Monsoma pulveratum (Retzius) (Hymenoptera: Tenthredinidae: Allantinae), a Palaearctic sawfly defoliator of alder in Alaska and new to the United States. Proceedings of the Entomological Society of Washington 112: 332-335. doi: 10.4289/0013-8797-112.2.332

Liston AD (1993) Taxonomy and host associations of west Palaearctic Kaliofenusa Viereck: leafmining sawflies on Ulmus (Hymenoptera: Tenthredinidae). Entomologist's Gazette 44: 45-54.

Looney C, Sheehan K, Bai B, Flowers R, Kohler G, LaGasa E, McKorney AE, Smith DR (2012) Distribution of a potential new forest pest, Monsoma pulveratum (Hymenoptera: Tenthredinidae), in the Pacific Northwest states. Northwest Science 86: 342-345. doi: 10.3955/046.086.0409

Macek J, Šípek P (2015) Azalea sawfly Nematus lipovskyi (Hymenoptera: Tenthredinidae), a new invasive species in Europe. European Journal of Entomology 112: 180-186.

MacGillivray AD (1920) Two new species of Platycampus (Hymenoptera Tenthredinidae). The Canadian Entomologist 52: 59-61. doi: 10.4039/Ent5259-3

MacNay CG (1964) First record of the sawfly Pristiphora aquilegiae (Voll.) in North America. Plant Protection 45: 91.

MacQuarrie CJK, Langor DW, Sperling FAH (2007) Mitochodrial DNA variation in two invasive birch leaf-mining sawflies in North America. Canadian Entomologist 139: 545-553. doi: $10.4039 / \mathrm{n} 06-084$

Mudge AD, LaBonte JR, Johnson KJR, LaGasa EH (2001) Exotic woodboring Coleoptera (Micromalthidae, Scolytidae) and Hymenoptera (Xiphydriidae) new to Oregon and Washington. Proceedings of the Entomological Society of Washington 103: 1011-1019.

Nealis VG (1991) Natural enemies and forest pest management. The Forestry Chronicle 67: 500-505. doi: 10.5558/tfc67500-5

Nielson MM, Morris RF (1964) The regulation of European spruce sawfly numbers in the maritime provinces of Canada from 1937-1963. The Canadian Entomologist 96: 773-784. doi: 10.4039/Ent96773-5

Pasteels JM, Gregoire J-C (1984) Selective predation on chemically defended chrysomelid larvae. Journal of Chemical Ecology 10: 1693-1700. doi: 10.1007/BF00987355

Powell JA, Turner WJ (1975) Observations on oviposition behavior and host selection in Orussus occidentalis (Hymenoptera: Siricoidea). Journal of the Kansas Entomological Society 48: 299-307.

Price PW (1970) A loosestrife sawfly, Monostegia abdominalis (Hymentopera: Tenthredinidae). Canadian Entomologist 102: 491-495. doi: 10.4039/Ent102491-4

Prous M, Blank SM, Goulet H, Heibo E, Liston A, Malm T, Nyman T, Schmidt S, Smith DR, Vårdal H, Viitasaari M, Vikberg V, Taeger A (2014) The genera of Nematinae (Hyme- 
noptera, Tenthredinidae). Journal of Hymenoptera Research (Special Issue), 69 pp. doi: 10.3897/JHR.40.7442

Quednau FW (1990) Introduction, permanent establishment, and dispersal in eastern Canada of Olesicampe geniculatae Quednau and Lim (Hymenoptera: Ichneumonidae), an important biological control agent of the mountain ash sawfly, Pristiphora geniculata (Hartig) (Hymenoptera: Tenthredinidae). The Canadian Entomologist 122: 921-934. doi: 10.4039/Ent122921-9

Raizenne H (1957) Forest sawflies of southern Ontario and their parasites. Canada Department of Agriculture Publication 1009, 45 pp.

Reeks WA, Barter GW (1951) Growth reduction and mortality of spruce caused by the European spruce sawfly, Gilpinia hercyniae (Htg.) (Hymenoptera: Diprionidae). The Forestry Chronicle 27: 140-156. doi: 10.5558/tfc27140-2

Ross HH (1955) The taxonomy and evolution of the sawfly genus Neodiprion. Forest Science 1: 196-209.

Scannell CM (2000) The Biology and Seasonal Life History of the Elm Leaf Miner, Fenusa ulmi (Sundevall), in the Pacific Northwest (Hymenoptera:Tenthredinidae). Masters Thesis, University Washington, Seattle.

Schaffner JV Jr (1936) European Pristiphora geniculata sawfly attacks mountain ash in the United States. Journal of Economic Entomology 29: 469.

Schaffner JV Jr (1939) Neodiprion sertifer (Geoff.), a pine sawfly accidentally introduced into New Jersey from Europe. Journal of Economic Entomology 32: 887-888.

Schaffner JV Jr (1943) Sawflies injurious to conifers in the northeastern states. Journal of Forestry 41: 580-88.

Schiff NM, Goulet H, Smith DR, Boudreault C, Wilson AD, Scheffler BE (2012) Siricidae (Hymenoptera: Symphyta: Siricoidea) of the Western Hemisphere. Canadian Journal of Arthropod Identification 21: 1-305.

Seattle Times (1996) Columbine Muncher is Likely Sawfly, Not Rabbit. http://community. seattletimes.nwsource.com/archive/?date $=19960920 \&$ slug $=2350147$

Sinclair WA, Lyon HH, Johnson WT (1987) Diseases of Trees and Shrubs. Cornell University Press, Ithaca, $574 \mathrm{pp}$.

Slingerland MV (1905) Two new shade-tree pests: sawfly leaf-miners on European elms and alder. Cornell University Agricultural Experiment Station Bulletin 233: 49-62.

Smith DR (1967) A review of the subfamily Heterarthrinae in North America (Hymenoptera: Tenthredinidae). Proceedings of the Entomological Society of Washington 69: 277-284.

Smith DR (1969) Nearctic Sawflies. I. Blennocampinae: Adults and larvae (Hymenoptera: Tenthredinidae). U.S. Department of Agriculture, Technical Bulletin No.1397, 179 pp.

Smith DR (1971) Nearctic Sawflies III. Heterarthrinae: Adults and larvae (Hymenoptera: Tenthredinidae). U. S. Department of Agriculture Technical Bulletin No. 1420, 84 pp.

Smith DR (1972) Sawflies of the genus Croesus Leach in North America. Proceedings of the Entomological Society of Washington 74: 169-180.

Smith DR (1974a) Sawflies of the tribe Cladiini in North America (Hymenoptera: Tenthredinidae: Nematinae). Transactions of the American Entomological Society 100: 1-28. 
Smith DR (1974b) Azalea sawflies and a new species of Nematus Panzer (Hymenoptera: Tenthredinidae). Proceedings of the Entomological Society of Washington 76: 204-207.

Smith DR (1979a) Symphyta. In: Krombein KV, Hurd, PD Jr., Smith DR, Burks BD (Eds) Catalog of Hymenoptera in America North of Mexico. Volume 1, Symphyta and Apocrita (Parasitica). Smithsonian Institution Press, Washington, 1-137.

Smith DR (1979b) Nearctic sawflies IV. Allantinae: Adults and larvae (Hymenoptera: Tenthredinidae). U. S. Department of Agriculture Technical Bulletin No. 1595, 172 pp.

Smith DR (1983) Xiphydria prolongata (Geoffroy) (Hymenoptera: Xiphydriidae) adventive in North America. Proceedings of the Entomological Society of Washington 85: 860-861.

Smith DR (1989) The sawfly genus Arge (Hymenoptera: Argidae) in the Western Hemisphere. Transactions of the American Entomological Society 115: 83-205.

Snyder C, MacQuarrie CJK, Zogas K, Kruse JJ, Hard J (2007) Invasive species in the last frontier: distribution and phenology of birch leaf mining sawflies in Alaska. Journal of Forestry 105: $113-155$.

Taeger A, Altenhofer E, Blank SM, Jansen E, Kraus M, Pschorn-Walcher H, Ritzau C (1998) Kommentare zur Biologie, Verbreitung und Gefährdung der Pflanzenwespen Deutschlands (Hymenoptera, Symphyta). In: Taeger A, Blank SM (Hrsg.) Pflanzenwespen Deutschlands (Hymenoptera, Symphyta). Kommentierte Bestandsaufnahme. Goecke \& Evers, Keltern, 49-135.

Taeger A, Blank SM, Liston AD (2010) World catalog of Symphyta. Zootaxa 2580: 1-1064.

Tsao CH, Hodson AC (1956) The effect of different host species on the oviposition and survival of the introduced pine sawfly. Journal of Economic Entomology 49: 400-401. doi: $10.1093 /$ jee/49.3.400

Williams, DJ (2007) Biology of the spiny ash sawfly, Eupareophora parca (Hymenoptera: Tenthredinidae: Blennocampinae), in Edmonton, Alberta. The Canadian Entomologist 139: 269-277. doi: 10.4039/n06-026

Wilson LF (1966) Introduced pine sawfly. US Department of Agriculture, Forest Service, Forest Insect and Disease Leaflet 99.

Wilson LF (1971) European pine sawfly. US Department of Agriculture, Forest Service, Forest Insect and Disease Leaflet 98.

Van Driesche RG, Healy S, Reardon RC (1996) Biological Control of Arthropod Pests of the Northeastern and North Central Forests in the United States: A Review and Recommendations. FHTET-96-19. USDA Forest Service, Forest Health Technical Enterprise Team, Morgantown.

Van Driesche RG, LaForest JH, Bargeron CT, Reardon RC, Herlihy M (2012) Forest Pest Insects in North America: a Photographic Guide. FHTET-2012-02. USDA Forest Service, Forest Health Technical Enterprise Team, Morgantown. 